\title{
On the Upper Ocean Thermal Structure in a Western North Pacific Ocean Model: Model Evaluation and Sensitivity Study
}

\author{
Mien-Tze Kueh ${ }^{1}$, Chung-Hsiung Sui ${ }^{1,2}, \mathrm{Kon}^{-K e e ~ L i u}{ }^{2, *}$, and Fei Chai ${ }^{3}$ \\ ${ }^{1}$ Department of Atmospheric Sciences, National Central University, Jhongli, Taiwan, ROC \\ ${ }^{2}$ Institute of Hydrological and Oceanic Sciences, National Central University, Jhongli, Taiwan, ROC \\ ${ }^{3}$ University of Maine, School of Marine Sciences, Maine 04469, USA
}

Received 25 September 2008, accepted 3 June 2009

\begin{abstract}
Seasonal variations in the upper water column of the western North Pacific are simulated with the Regional Ocean Model System (ROMS). The model is driven by surface fluxes of heat, momentum and freshwater without prescribing sea surface temperature or salinity. A series of numerical experiments are conducted to explore the sensitivity of the upper ocean thermal structure to the parameterized solar penetration scheme and two common practices to control model climate through a flux correction term and a nudging term. The absorption of solar radiation by the water column beneath the sea surface destabilizes the upper layers to cause a stronger mixing and deeper mixed layer in the warm season (from April to September). Therefore, removing solar penetration from the model results in an exceptionally stable surface layer, and tends to produce an overly shallow mixed layer in the warm season. The experiment with a prescribed net surface heat flux shows that the model is unable to maintain a heat balance in the upper water column, producing a cooling trend. Experiments with a flux correction term are able to keep the simulated sea surface temperature (SST) from a long term drift by adjusting the amount of the net surface heat flux. However, unrealistic net surface heat flux is produced in the experiment, when the model assumes no solar penetration. The implementation of a weak temperature nudging (1/50 days) toward a long term mean climatology prevents the model from simulating a cold bias during long term integration. The experiment with solar penetration and a weak nudging produces reasonable interannual variability during the period of 1995 - 2006 without flux corrections. The nudging terms steer advective heat fluxes towards the climatological mean state so as to avoid long-term drift in upper water column heat content. A detailed understanding of the function of nudging terms in controlling the hydrodynamics of the water column remains to be investigated.
\end{abstract}

Key words: ROMS, Upper ocean thermal structure, Western North Pacific, Solar penetration, Interannual variation, Model sensitivity Citation: Kueh, M. T., C. H. Sui, K. K. Liu, and F. Chai, 2010: On the upper ocean thermal structure in a western North Pacific Ocean model: Model evaluation and sensitivity study. Terr. Atmos. Ocean. Sci., 21, 137-162, doi: 10.3319/TAO.2009.06.03.01(IWNOP)

\section{INTRODUCTION}

The ocean is a dominant component determining the Earth's heat balance. Based simply on the physical properties of water and air, namely the specific heat and density, Rossby (1959) suggested that ocean heat content dominates and contributes substantially to the climate variability. Since then, ocean models have been developed to simulate natural and anthropogenic climate changes. Many models have succeeded in simulating an El Niño evolution since the Tropical Ocean - Global Atmosphere (TOGA) era (e.g., Guilyardi

\footnotetext{
* Corresponding author

E-mail:kkliu@ncu.edu.tw
}

et al. 2009 and references therein). In recent decades, ocean models are increasingly dependent upon research for studying the earth response to increasing greenhouse warming. The radiative imbalance of the Earth's climate system can be diagnosed by the change in the heat content of the ocean (e.g., Pielke 2003). Observational studies have shown that the heat content of the upper ocean has been increasing over the last 48 years in the world's oceans (Levitus et al. 2000; Levitus et al. 2005). Observed ocean heat content changes have been reproduced by both a forced ocean model (Barnett et al. 2001) and coupled global climate model (Hansen et al. 2005). Both models are driven by anthropogenic factors, such as concentrations of greenhouse gases and the 
effect of aerosols in the atmosphere. This suggests that anthropogenic forcing could be responsible for the observed changes in heat content of the global oceans.

Despite successful simulations on interannual and long-term changes of the ocean's heat content, simulation of a detailed annual cycle in ocean models, especially regional models, is still a challenge. The most significant change in the upper ocean thermal structure is the seasonal variation in the stratification resulting from annual surface forcing. The variations of upper ocean thermal structure are closely related to the evolution and maintenance of the oceanic surface mixed layer, which involves momentum and heat transfer across the air-sea interface and turbulent mixing both in the atmospheric and oceanic boundary layers. To accurately simulate these processes, which are not completely understood yet, is often difficult.

In order to better simulate an upper ocean thermal structure, we may employ various thermal boundary conditions for the upper boundary in ocean models. Perhaps the simplest (but also the crudest) upper thermal boundary condition is to force the model by prescribing the sea surface temperature and salinity with observed values. An alternative is to restore the sea surface temperature and salinity toward observed values on a specific timescale to avoid over-constraint on model solutions (Sarmiento and Bryan 1982; Marotzke and Willebrand 1991; Weaver and Sarachik 1991). Such an approach computes the surface heat flux with a restoring boundary condition, which is expressed as a form of the difference between simulated and observed sea surface temperature. Under this kind of upper boundary condition, large heat fluxes can only occur if the sea surface temperature differs significantly from the restoring temperature. Recently, an adjusted surface heat flux boundary conditions, which was first proposed by Haney (1971), has been widely used in forced ocean models (Oey and Chen 1992; Kagimoto and Yamagata 1997; Ezer 2000; Haidvogel et al. 2000, 2008). This type of upper boundary conditions adopts a so-called flux correction term to the surface heat flux used in the model. A variety of the coefficient of the flux correction term is used, ranging from a specific value of restoring timescale (Oey and Chen 1992; Kagimoto and Yamagata 1997) to the sensitivity of the surface heat flux to sea surface temperature with spatial and temporal variations (Haidvogel et al. 2000, 2008).

The importance of solar irradiance penetration on sea surface temperature and mixed layer depth evolution has long been illustrated by one-dimensional mixed layer modeling studies (e.g., Martin 1985; Large et al. 1994). The penetrating solar irradiance is regulated primarily by the magnitude of the incident solar irradiance and the variation of optical attenuation in the upper ocean, which is commonly attributed to changes in chlorophyll distribution (e.g., Lewis et al. 1990; Siegel et al. 1995). There was a variety of solar penetration parameterizations for modeling mixed layer radiant heating. For example, Paulson and Simpson (1977) proposed a commonly used Jerlov water-based parameterization that expressed solar penetration as a function of a Jerlov water type. The Jerlov water type was developed as a proxy for different patterns of vertical chlorophyll distribution (Jerlov 1976). More recently, studies on implementation of chlorophyll-dependent solar irradiance penetration parameterization on ocean general circulation models revealed the benefit of the spatial and temporal variations in solar irradiance penetration depth, which not only improved the simulation of sea surface temperature and upper ocean stratification (Ohlmann 2003) but also influenced ocean heat transport (Sweeney et al. 2005).

In addition to upper boundary conditions, relaxation of a simulated temperature is also applied to prevent the model results from climate drift. The model interior temperature is relaxed toward a reference value, commonly the observed climatology, by adding a nudging term to the temperature equation (Sarmiento and Bryan 1982; Oey and Chen 1992; Kagimoto and Yamagata 1997). Thus the variability in the model shall be constricted by the given nudging coefficient, which is often a timescale of several tens of days.

The goal of this study is to construct a regional ocean model to study the upper ocean thermal structure in the western North Pacific Ocean, and to test the sensitivity of the upper ocean thermal structure to some commonly used assumptions in upper boundary conditions for large-scale ocean model. The model results are evaluated mainly in terms of seasonal variations. The paper is organized as follows. The ocean model configuration, the experiment design and the surface forcing are described in section 2 . The results of different model experiments are compared with observations in section 3. Finally, a discussion and summary are given in section 4 .

\section{MODEL DESCRIPTION AND EXPERIMENT DE- SIGN}

The western North Pacific Ocean model used in the study is developed based on the Regional Ocean Modeling System (ROMS, version 2.2). The ROMS is a three dimensional, free-surface, finite difference model based on the generalized nonlinear terrain-following coordinate of Song and Haidvogel (1994). The nonlinear stretching of the vertical coordinate, which depends on local water depth, can be configured to generate enhanced resolution near either the sea surface or sea floor. The ROMS is a modern ocean model system with several new features, including alternatives for high-order upstream-biased advection, and for subgridscale parameterization. A brief description of the ROMS and the model evaluation is given in Haidvogel et al. (2000). A more detailed description of the discretization and numerical algorithms for the ROMS can be found in Haidvogel et al. (2008). 


\subsection{Model Configuration}

Figure 1 shows the model domain and the bottom topography. The horizontal grid has uniform spacing on a Mercator's projection with a resolution of $0.5^{\circ}$ approximately in the zonal direction, and various resolutions ranging from $0.48-0.15^{\circ}$ in a south-north direction (with $\Delta y$ getting smaller as the grid stretches farther from the equator). The grid was chosen to extend from about $0.9^{\circ} \mathrm{S}$ to $45^{\circ} \mathrm{N}$, and 99 to $160^{\circ} \mathrm{E}$. The bathymetry was derived from the ETOPO5 (5 min) gridded dataset (National Geophysics Data Center 1988). The vertical stretching sigma grid in the study has 20 levels; the stretched s-coordinate parameters $(\theta=5$ and $\left.\theta_{b}=0.4\right)$ are set according to Haidvogel et al. (2000), so as to obtain a higher resolution in the upper mixed layer. A cross section of the vertical coordinate surfaces along $15^{\circ} \mathrm{N}$ is shown in Fig. 2. The deepest bottom topography in the model is set to $5500 \mathrm{~m}$; at this depth, there are five layers in the upper $100 \mathrm{~m}$, and proportionally higher vertical resolution is obtained in shallower regions (the shallowest depth in the model is set to $10 \mathrm{~m}$ ). For a terrain-following coordinate system, there is a systematic error associated with the possibility of significant pressure gradient errors, which depend on the steepness of the topography, the horizontal and vertical resolutions, and the strength of the stratification. The method for computing horizontal pressure-gradient force introduced by Shchepetkin and McWilliams (2003), which has been implemented to the ROMS version 2.2 , is selected in our work to account for the possible errors. After interpolation, the topography was smoothed using a Shapiro-based filter in regions where the $\mathrm{r}$-value exceeds a specific value. The r-value is a parameter introduced by Beckmann and Haidvogel (1993), which is applied to ensure stable and accurate simulation using realistic bathymetry. Various $\mathrm{r}$-values have been tested, and a value of 0.25 is used for experiments in this study. Furthermore, a special treatment is also conducted to retain some small island arcs, continental shelves and the deepness of some straits by lifting/lowering the sea floor back to their origin depths based upon a set of specific depths in between the smoothing of the bathymetry. Our sensitivity tests have shown that the continental slope can be an important factor in control of the non-seasonal depth-averaged transport of the Kuroshio.

The southern, eastern, and northern boundaries are open except for a few landmasses. The western boundary is closed. The South China Sea is open to the southern boundary and connected to the open ocean through several passages between the surrounding landmasses and islands which include the Luzon Strait and Taiwan
Strait. The Strait of Malacca is closed, while the Makassar Strait and Maluku Strait are open along the southern boundary. The Sea of Japan is open to the northern boundary and connected to the East China Sea and the Pacific through the Tsushima Straits and Tsugaru Strait, respectively. The radiation boundary condition and a buffer zone are applied to all open boundaries. In these nudging zones (about $3^{\circ}$ wide), temperature, salinity, and baroclinic horizontal velocities are relaxed to their observed monthly climatology. The monthly climatological temperature and salinity are derived from the NODC World Ocean Atlas 1998 (WOA98, the Levitus set), while the baroclinic horizontal velocities are drawn from the Simple Ocean Data Assimilation (SODA version 3.4, Carton et al. 2000a, b) The monthly climatology of SODA is obtained from a long term mean dating from 1950 - 2001. The nudging/relaxation time scale is 5 days at the domain boundaries increasing to 50 days at the interior edge of the buffer zone. The factor between the passive and active open boundary conditions is 15 . Free-slip lateral boundary conditions are applied on all solid sidewalls.

The third-order upstream (TOUS) advection scheme of Shchepetkin and McWilliams (1998) is used. This advection scheme is associated with weak, fourth-order (bi-harmonic) smoothing. Thus no explicit horizontal dissipation or diffusion is needed. Vertical mixing is determined by the KPP scheme (Large et al. 1994). Tides and river discharges are omitted in the model. The baroclinic and barotropic timesteps are 1800 and $30 \mathrm{~s}$, respectively (i.e., there are 60 barotropic time steps per baroclinic step).

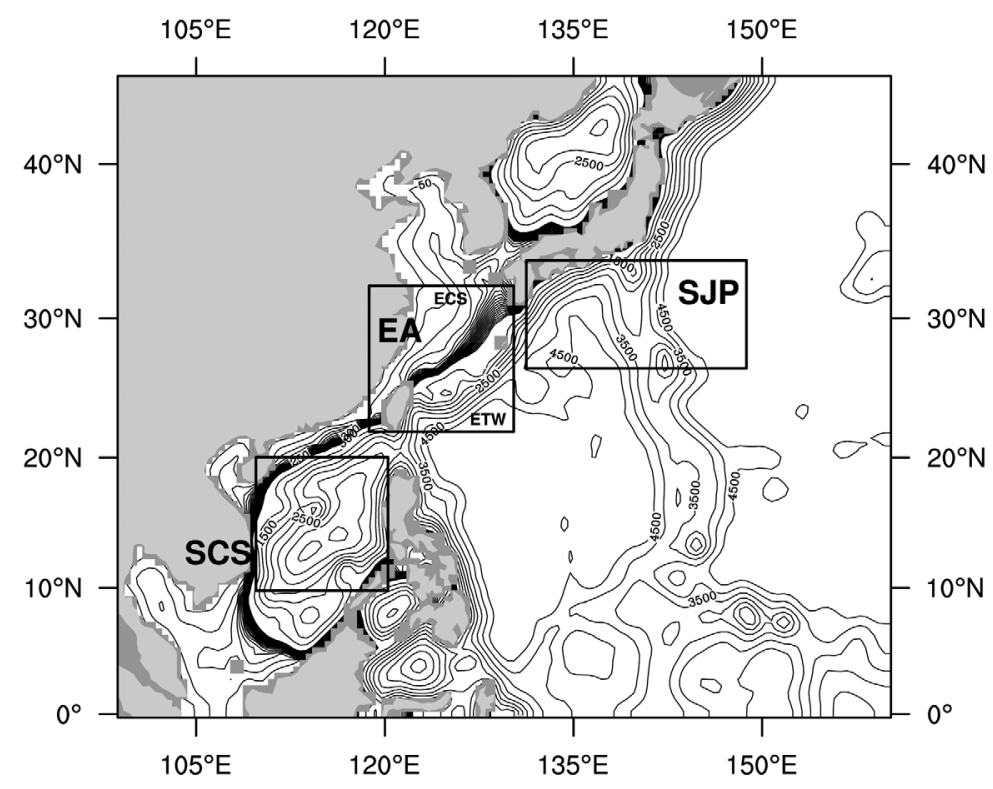

Fig. 1. Model domain and bottom topography (in meters). The contour interval is $25 \mathrm{~m}(500 \mathrm{~m})$ for the water column depths less than (exceed) $500 \mathrm{~m}$. The minimum and maximum depths are artificially fixed at $10 \mathrm{~m}$ and $5500 \mathrm{~m}$, respectively. The three boxed regions $S C S, E A$, and $S J P$ are used for analysis as described in the text. The sub-regions in $E A, E C S$ and $E T W$, are defined by grids of water depths less than $100 \mathrm{~m}$ and exceeding $500 \mathrm{~m}$, respectively. 


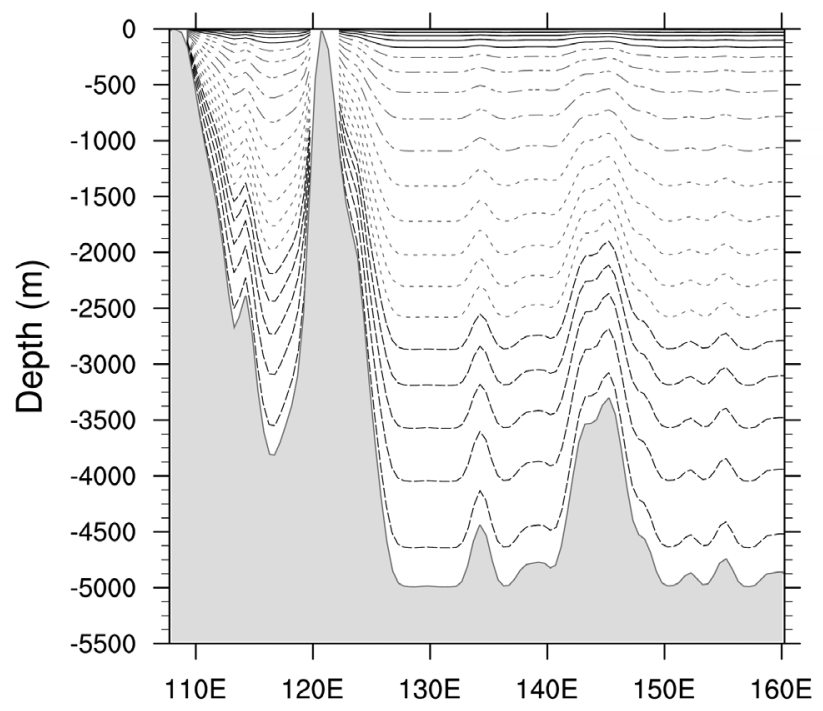

Fig. 2. Section along $15^{\circ} \mathrm{N}$ showing vertical coordinate surfaces used in the experiments. The vertical resolution is 20 sigma levels, and the stretched s-coordinate parameters are set to $\theta=5$ and $\theta_{b}=0.4$, respectively.

\subsection{Experiment Design}

The experiments designed for the study is according to the tendency equation of potential temperature in the ROMS:

$\frac{\partial\left(H_{z} T\right)}{\partial t}+\frac{\partial\left(u H_{z} T\right)}{\partial x}+\frac{\partial\left(v H_{z} T\right)}{\partial y}+\frac{\partial\left(w H_{z} T\right)}{\partial s}=H_{z}\left(D_{T}+F_{T}\right)(1)$

where the $T$ is the potential temperature; $u, v$, and $w$ are the components of velocity in the horizontal ( $x$ and $y)$ and vertical (scaled sigma coordinate, $s$ ); $H_{z}$ is a vertical stretching factor. The diffusive term $D_{T}$ can be parameterized as $-K_{H} \partial \rho / \partial z$, here the eddy diffusivity $K_{H}$ is calculated using the KPP scheme. The forcing term $F_{T}$ applied on the upper boundary condition can be prescribed as follows:

$F_{T}=\rho_{w} C_{p w}\left[Q^{*}+Q_{s}+\frac{d Q}{d S S T_{r e f}}\left(S S T-S S T_{r e f}\right)\right]$

Here $Q$ is the net surface heat flux $\left(Q=Q_{s}+Q^{*}\right), Q_{s}$ is the shortwave radiation, and $Q^{*}$ is the sum of surface heat fluxes of sensible, latent, and longwave radiation. The SST and $S S T_{r e f}$ are the model and reference sea surface temperature, respectively. The $\rho_{w}$ and $C_{p w}$ are the sea water density and the specific heat of water, respectively. The last term on the right-hand side of Eq. (2) is a flux correction term which is derived based on the pioneering work of Haney (1971) wherein he used the near surface air temperature as a reference surface temperature in the formula to derive the net surface heat flux, assuming that the observed air tem- perature is relatively more reliable than the heat flux. The idea of the Haney-type formulation in Eq. (2), is to express a feedback to the atmosphere as a flux correction which depends upon the model SST, and partly compensates for errors in the observed heat flux. The $d Q / d S S T_{\text {ref }}$, which is treated as a "damping coefficient" with spatial and monthly variability, is obtained from the Comprehensive OceanAtmosphere Data Set (COADS) monthly climatology (da Silva et al. 1994) in the study. The reference sea surface temperature $S S T_{\text {ref }}$ is also derived from the COADS monthly climatology. The flux correction term is only applied for selected experiments.

Two additional terms, the shortwave radiation penetration term and the nudging term, are added to the right hand side of Eq. (1) for several selected experiments. The penetrative portion of the shortwave radiation that is absorbed beneath the surface is parameterized by the least-squares fit expression given by Paulson and Simpson (1977):

$I(z)=I_{0}\left[\operatorname{Re}^{z / \zeta_{1}}+(1-R) e^{z / \zeta_{2}}\right]$

where the $I(z)$ is the penetrative portion of shortwave radiation as a function of depth $(z)$, and $I_{0}$ is the surface irradiance (namely, the $Q_{s}$ ). $R$ is the empirical fractional coefficient for this two-band model; the attenuation lengths $\zeta_{1}$ and $\zeta_{2}$ are preferential band absorption coefficients for the infrared and red wavelengths in the upper $5 \mathrm{~m}$ and the shorter blue-green light below 10 m, respectively. Martin (1985) indicated that the modeled mixed layer depth (MLD) and surface temperature are quite sensitive to the choice of the water type, though here only one water type is used for the entire domain. It is noted that the term $Q_{s}$ in Eq. (2) should be replaced by $I(z)$ when the solar penetration term is applied. Diurnal cycling is applied to all the experiments in our study. The nudging term is expressed as:

$-\gamma\left(T-T_{r e f}\right)$

where $\gamma$ is the nudging coefficient, which is set to the inversed value of 50 days, for relaxing the potential temperature $(T)$ toward the reference temperature $\left(T_{r e f}\right)$ for the entire model interior. The three dimensional reference temperatures were drawn from the WOA98 climatology.

The flux correction term, the shortwave radiation penetrative term, and the nudging term will be applied selectively to conduct various experiments in the study. Furthermore, a similar freshwater flux correction is also applied selectively to the salinity equation. The emphasis herein is on the influence of the aforementioned terms on the simulated upper ocean thermal structure.

The experiments in the study follow a spinup of 5-year simulations starting from the initial conditions derived from the January temperature and salinity of the WOA98 clima- 
tology, and baroclinic horizontal velocities of the SODA v3.4 climatology. Surface forcing during the spinup period is the monthly climatological wind stress, heat flux(es), and freshwater flux from the COADS. The spinup simulation is performed using all the aforesaid terms. Two groups of experiments, the climatological group and interannual group have been performed hereafter (Table 1). The former is conducted using the surface forcing from the COADS climatology, and the later using the monthly surface fluxes from NCEP during 1995 - 2006. To emphasize the role of the upper boundary conditions in the interannual run, the lateral boundary conditions herein have been held fixed as the climatological run as depicted in the previous section.

Seven experiments are conducted within the climatological group. All experiments start from the last output fields of the spinup simulation and integrate for 5-year. Table 1 summarizes the attributes of the seven experiments, which are designed according to the degree of freedom from constraints on surface processes. The first experiment has the same setting as that of the spinup simulation by retaining all four terms (heat flux correction term, freshwater flux correction term, the penetrative shortwave radiation term, and the nudging term), and is denoted as the control experiment (CNTL in Table 1). The heat flux correction term is dropped in the experiment noQC, while both the heat flux correction and freshwater flux correction are abandoned in the experiment noFC. The penetrative shortwave radiation term and the nudging term are removed in the experiments noSP and noCndg, respectively. The above four experiments are designed for examining the relative effect of the terms on temperature simulation. Experiment SP is conducted retaining the penetrative shortwave radiation term only, and experiment Cndg is performed retaining the nudging term solely. The two experiments are designed to assess the respective impact of the corresponding term. A nudging term for the baroclinic momentum fields is also applied to the five experiments with the imposed temperature nudging term, namely the experiments CNTL, noQC, noSP, noFC, and Cndg. Additional experiments to evaluate the sensitivity of the model to the nudging toward momentum climatology will be briefly described later. It should be noted that, under the experiment design here, no prescribed sea surface temperature, salinity, or height are used.

Of the two experiments in the interannual group, the first applies the setting of experiment noFC, and the second adopts that of experiment SP. Both interannual experiments are started from the last model outputs of experiment noFC, and integrated for 12 years.

All seven experiments in the climatological group are evaluated based on the 5-year simulations against the WOA98 monthly climatology, while the two interannual experiments are evaluated using the optimum interpolation (OI) sea surface temperature (SST) analysis (Reynolds and Smith 1994; Reynolds et al. 2002). In particular, analyses of the thermal structures and turbulence parameters are focused on properties averaged over four regions, the South China Sea $(S C S)$, the region to the east of Taiwan $(E T W)$, the East China Sea $(E C S)$, and the region to the south of Japan $(S J P)$, as denoted in Fig. 1. Note that ECS and ETW are combined to form the region $E A$ in Fig. 1 wherein they are defined by grids of water depths less than $100 \mathrm{~m}$ and exceeding $500 \mathrm{~m}$, respectively. The regions $E T W$ and $S J P$ are chosen to represent the Kuroshio and Kuroshio Extension regions, respectively.

\subsection{Surface Forcing}

The seasonal changes of the surface heat fluxes and radiation averaged over the three boxed region in Fig. 1 are shown in Fig. 3a. For region $E A$ only the area-averaged values are shown without further dividing it into subregions of $E C S$ and $E T W$, since their values are quite similar, in terms of the magnitudes and changing rates. The differences between $E A$ and $S J P$ are also very little. The $E A$ and $S J P$ exhibit significant seasonal variations with a period of heating to the ocean in the warm season (from April to September).

Table 1. Two groups of experiments performed in this study to test the effect of thermal forcing and corrections in the model on upper ocean thermal structure. The climatological group consists of seven experiments integrated for five years starting from the end of a five-year spinup run. The interannual group consists of two experiments integrated for twelve years starting from the end of noFC experiment. See text for details.

\begin{tabular}{|c|c|c|c|c|c|c|c|c|c|}
\hline & \multicolumn{7}{|c|}{ CLIMATOLOGICAL RUN } & \multicolumn{2}{|c|}{ INTERANNUAL RUN } \\
\hline & CNTL & noQC & noSP & noFC & noCndg & Cndg & SP & year_noFC & year_SP \\
\hline Heat flux correction & $\mathrm{v}$ & & $\mathrm{v}$ & & $\mathrm{v}$ & & & & \\
\hline Freshwater flux correction & $\mathrm{v}$ & $\mathrm{v}$ & $\mathrm{v}$ & & $\mathrm{v}$ & & & & \\
\hline Shortwave radiation penetration & $\mathrm{v}$ & $\mathrm{v}$ & & $\mathrm{v}$ & $\mathrm{v}$ & & $\mathrm{v}$ & $\mathrm{v}$ & $\mathrm{v}$ \\
\hline Nudging term & $\mathrm{v}$ & $\mathrm{v}$ & $\mathrm{v}$ & $\mathrm{v}$ & & $\mathrm{v}$ & & $\mathrm{v}$ & \\
\hline
\end{tabular}


(a)
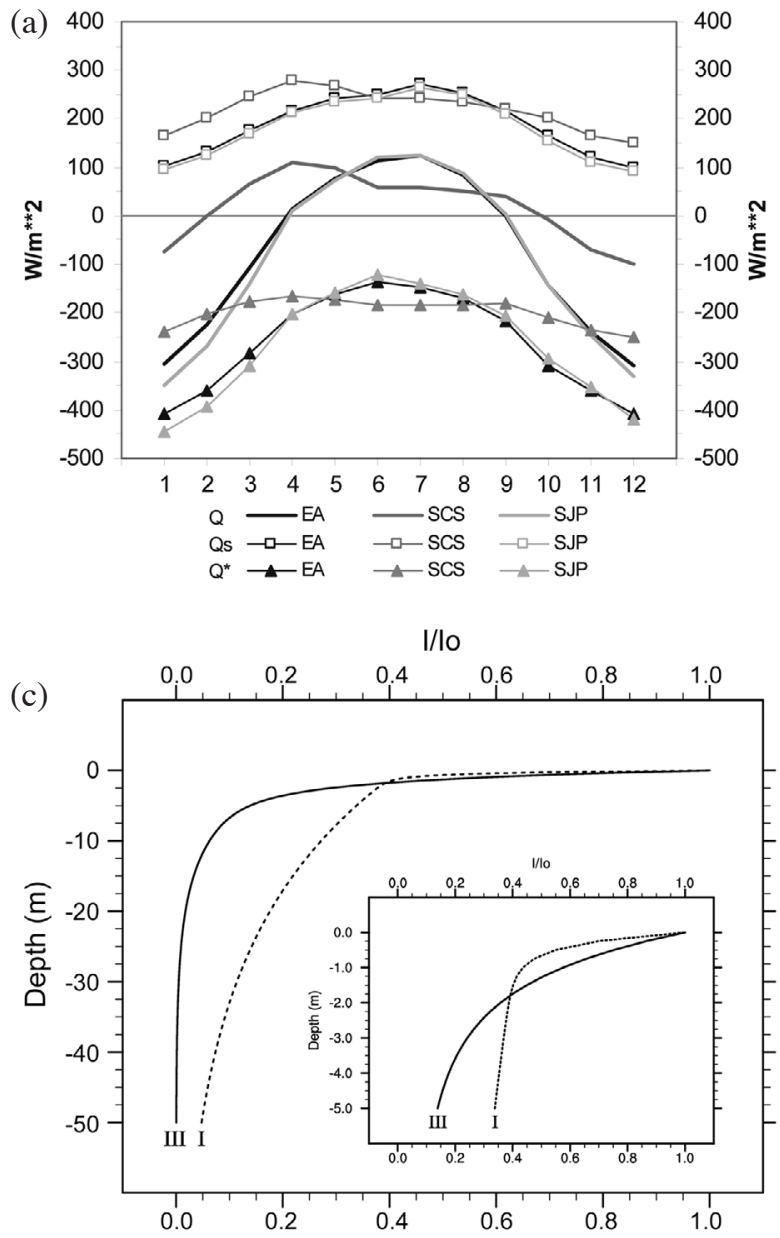

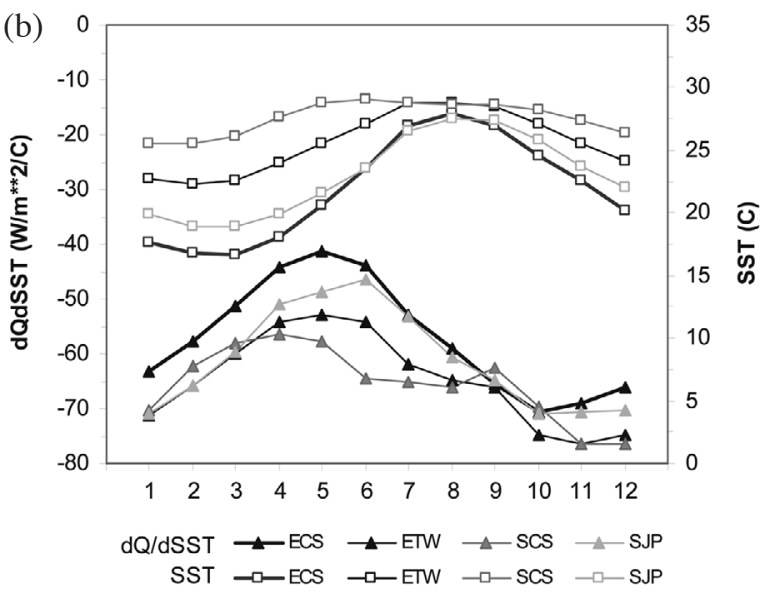

Surface wind stress $\left(10 \mathrm{E}-2 \mathrm{~N} / \mathrm{m}^{* *} 2\right)$

(d)

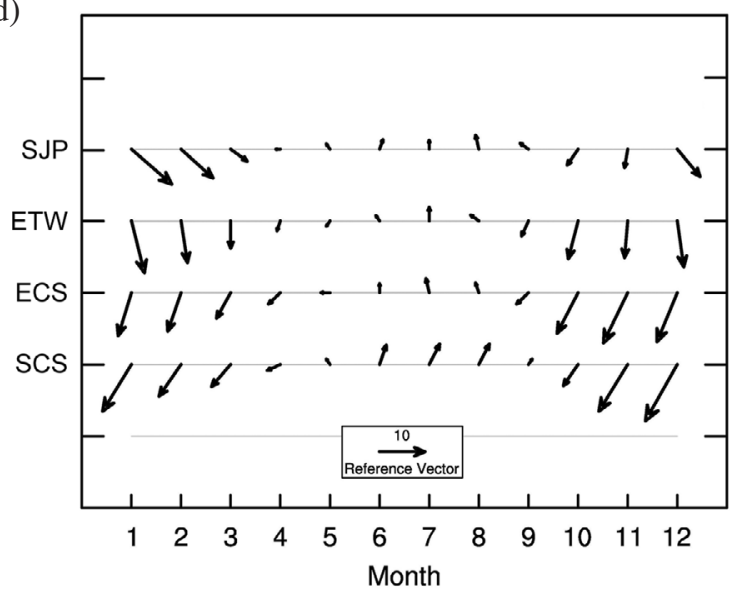

Fig. 3. (a) The area-averaged net surface heat flux excluding the shortwave radiation $\left(Q^{*}\right.$, thin lines with filled triangles), the incoming shortwave radiation $\left(Q_{s}\right.$, thin lines with hollow squares), and the net surface heat flux $\left(\mathrm{Q}=Q_{s}+Q^{*}\right.$, thick lines). The unit is "W $\mathrm{m}^{-2}$ ", positive values represent heating of the ocean. (b) The area-averaged sea surface temperature ( $S S T$ in Celsius; lines with hollow squares) and $Q$ sensitivity to $S S T_{\text {ref }}$

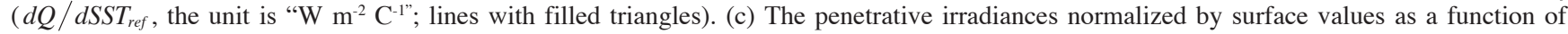
depths. The inset is the blow-up of the top $5 \mathrm{~m}$. The solid and dashed lines represent the normalized penetrative irradiances in water types I and III, respectively. The equation and values of parameters are given in text. (d) The area-averaged wind stresses ( $\left.\mathrm{N} \mathrm{m}^{-2}\right)$. The variables for (a), (b), and (d) are derived from climatological monthly mean COADS. The averaged areas are the three boxed regions in Fig. 1. The ECS and ETW represent subregions in region $E A$ with water depths less than $100 \mathrm{~m}$ and exceeding $500 \mathrm{~m}$, respectively.

On the other hand, the $S C S$ presents relatively moderate seasonality with a longer period of heating toward the ocean. The annual average net surface heat flux for the entire domain, EA, SCS, SJP are $-19.8,-76.4,19.3$, and $-88.0 \mathrm{~W} \mathrm{~m}^{-2}$, respectively. Only the southern region, such as SCS, shows a total heating of the ocean surface annually. Moreover, the net surface heat flux excluding the shortwave radiation are negative for all seasons, indicating a cooling of the ocean surface due to net surface heat flux. Overall, while the ocean is heating up by the shortwave radiation for all seasons, there is an annual cooling of the ocean surface on this time scale.

Figure $3 \mathrm{~b}$ shows the reference sea surface temperature and sensitivity of net heat flux to SST for the four averaged regions. They are the last term on the right side of Eq. (1). The area-averaged values of ECS and ETW are shown here, because the differences of $S S T_{r e f}$ and $d Q / d S S T_{\text {ref }}$ between the two subregions are more pronounced than those of the surface heat fluxes and radiation, implying that a smaller scale of spatial variations in these two fields. The $d Q / d S S T_{\text {ref }}$ for each region presents a peak value prior to the maximum of the heating as shown in Fig. 3a, while a lag of the $S S T_{\text {ref }}$ peak value is found. Climatologically, larger "corrective coefficients" occur during the transition period in the spring season, and warmer sea surface temperatures appear after the maximum heating period of the ocean (at least for these regions).

For the five experiments using the penetrative shortwave radiation term (Table 1), the values of the empirical attenuation coefficients $R, \zeta_{1}$, and $\zeta_{2}$ are set to $0.58,0.35$, and $23 \mathrm{~m}$ as suggested by Paulson and Simpson (1977) cor- 
responding to the water type I classified by Jerlov (1976). Figure $3 \mathrm{c}$ presents the normalized penetrative irradiance $[I(z)$ from Eq. (3)] as a function of depths. The normalization is derived using the incoming surface values $\left(I_{0}\right)$. For water type I, though about half of the incoming shortwave radiation is absorbed in the top $2 \mathrm{~m}$, still a substantial amount of the solar radiation penetrates to the water column beneath. Thus the penetrative term is expected to have significant influence on the structural change of temperature field. An additional experiment using water type III is also conducted for discussing the influence of different water types on the simulation of the upper ocean thermal structure. Compared to water type I, water type III absorbs more shortwave radiation in the top $2 \mathrm{~m}$, resulting in less heat energy transmitted to water below (Jerlov 1976). The area-averaged monthly surface wind stresses for the four regions are shown in Fig. 3d. The wind stresses is stronger and weaker in the cold and warm season, respectively. From the north (SJP) to the south $(S C S)$, the winter monsoon exhibits a transition from northwesterlies to northeasterlies. The magnitude of the summer monsoon is decreasing gradually from south toward the north, associated with southwesterlies to southeasterlies.

\section{RESULTS}

\subsection{Overview}

The time series of volume-integrated kinetic energy per unit area (also termed column integrated here) for all experiments are first examined in Fig. 4a. These time series show a quasi-equilibrium oscillation with a semi-annual cycle for most experiments except SP, noCndg, and year_SP that are not constrained by nudging for temperature and horizontal flow. For the latter three, drifts in water properties occur.

For experiments in the climatological group, the nudging terms effectively maintain the model at an equilibrium value of kinetic energy around $1980 \mathrm{~J} \mathrm{~m}^{-2}$, and the amplitude of semi-annual cycles at $100-200 \mathrm{~J} \mathrm{~m}^{-2}$. The differences among these experiments are insignificant as compared to temporal fluctuation. Without nudging, SP and noCndg exhibit a rapid rise in kinetic energy in the first half year, and then a sharp decrease until the last two years of the integration. No semiannual variations appear in these two experiments. The existence of the semiannual variation in ocean currents in the western tropical Pacific has also been reported by Qu et al. (2008) in an analysis of altimeter data combined with a high-resolution general circulation model wherein they reported the semiannual variations to local Ekman pumping along with the westward propagation of Rossby waves originating in the central tropical Pacific.

As for the interannual group, year_SP reaches a quasi equilibrium value of about $1400 \mathrm{~J} \mathrm{~m}^{-2}$ in the last five years of the integration. On the other hand, year_noFC exhibits a moderate increase in kinetic energy during the first three years of integration, followed by a smaller quasi semiannual fluctuation about an equilibrium value of about $2110 \mathrm{~J} \mathrm{~m}^{-2}$.

The time series of the volume-averaged temperature for all experiments are shown in Fig. 4b. The series exhibit a distinct annual cycle instead of a semi-annual cycle found in the flow field. The simulated mean temperatures in the experiments are quite similar to each other except SP, year_SP, and Cndg. In Cndg, summer temperature is highest among all experiments. However, the deviation of Cndg is much smaller than the amplitude of the seasonal variation in the time series. In SP and year_SP that contain solar penetration term solely without flux correction and nudging terms, the model experiences an apparent cooling tendency. Note that the rate of temperature drift in year_SP slows down by year 2002 , and the temperature reaches an equilibrium value in the last five years of the integration.

Figure $4 \mathrm{c}$ shows the differences between the control experiment and all other experiments. There are relatively large negative deviations in kinetic energy for SP, year_SP and noCndg; and cold departures of volume-averaged temperature for SP and year_SP. Although SP and noCndg present similar long-term drift in kinetic energy, only SP exhibits significant temperature trend. It is noted that the major difference between these two experiments is that the latter contains flux corrections. Discussion on this will be given in next section. Only Cndg presents a warm departure as compared with the control experiment, this warm departure is much larger in the SST field. Furthermore, the differences of $S S T$ for the other experiments are also larger than the volume-averaged temperature. These results indicate that the experiments design herein should be suitable for investigating the upper ocean thermal structure. There are some slight differences in the salinity among these experiments, revealing that those simulated salinity are also affected by the experimental settings. However, the magnitudes of the salinity differences are relatively small and will not be discussed here. Last, all experiments exhibit larger potential energy than the control experiment, except noCndg. Since the mass is conserved in the integration, changes in potential energy imply changes in stratification of the simulated ocean.

Both momentum and scalar fields show similar characteristics in the five years of climatological simulations. Thus only results from the last year integration are presented, unless noted otherwise.

The near surface horizontal velocities for the control experiment are presented in Fig. 5. The distribution of the South Equatorial Current (SEC) near the southern boundary and the weaker North Equatorial Current (NEC) over 8 $15^{\circ} \mathrm{N}$, with the North Equatorial Countercurrent (NECC) in between, are reasonably simulated. The NEC bifurcates off Mindanao around $13^{\circ} \mathrm{N}$ with a seasonal shift; the summer bifurcation latitude shifts slightly southward than that of 

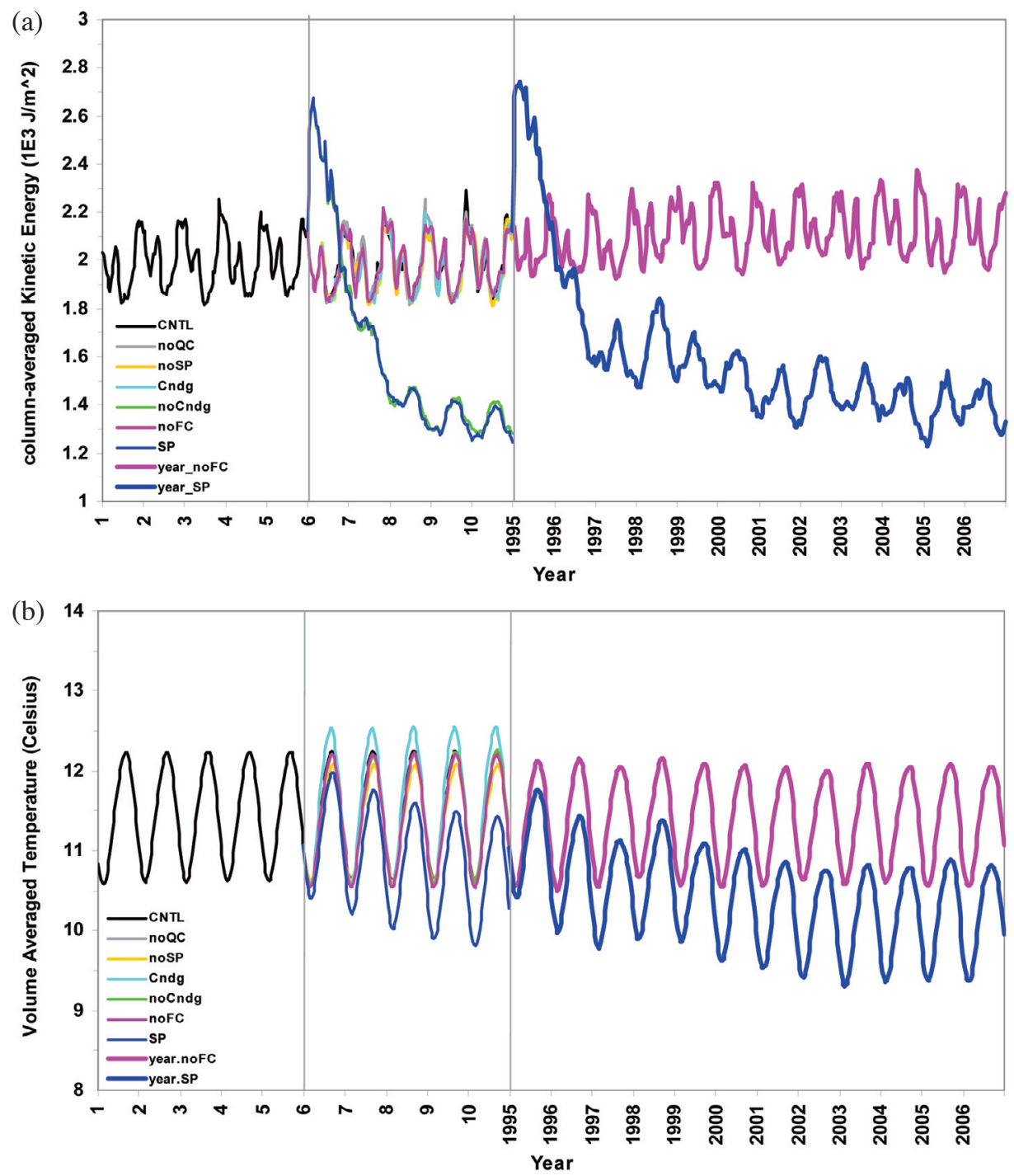

(c)

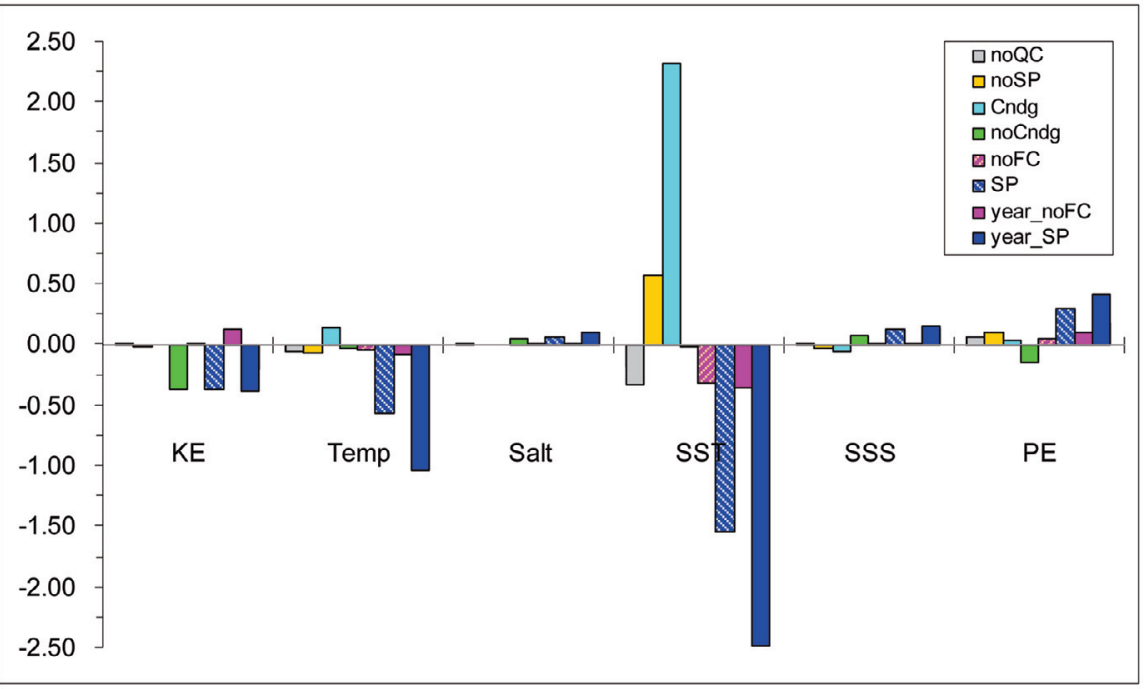

Fig. 4. Time series of (a) the column-averaged kinetic energy $\left(10^{3} \mathrm{~J} \mathrm{~m}^{-2}\right)$ and (b) the volume-averaged temperature (in Celsius). The two vertical grey lines on each panel indicate the start of year 6 and 1995. The lower panel (c) is the differences for various variables between the control experiment (CNTL) and all the other experiments. The derived differences are taken from variables including column-averaged kinetic energy (KE), temperature (Temp), salinity (Salt, in PSU), sea surface temperature (SST), sea surface salinity (SSS), and column-averaged potential energy (PE, in $\left.10^{7} \mathrm{~J} \mathrm{~m}^{-2}\right)$. The column-averaged means the volume-integrated values divided by surface area. 
(a)

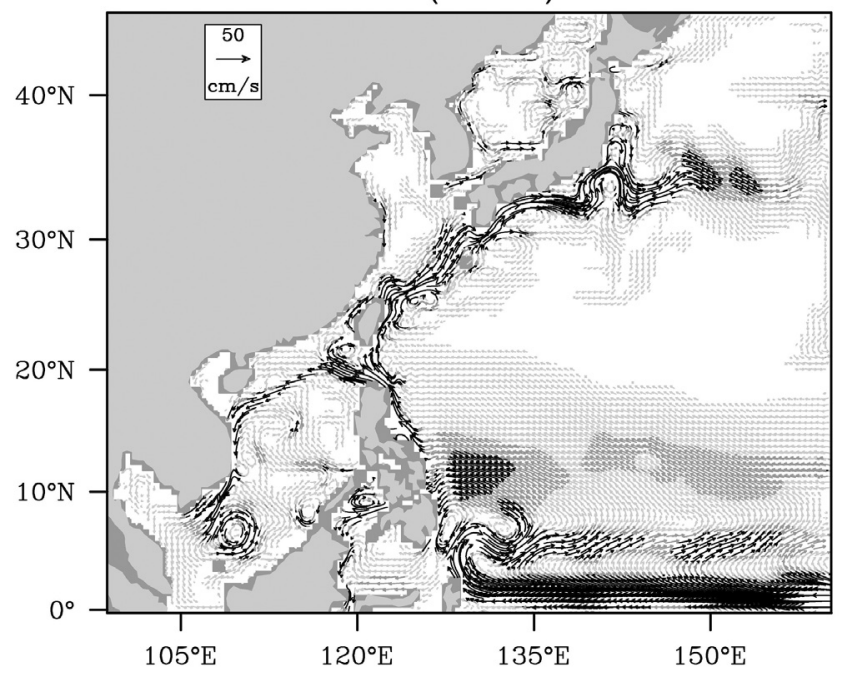

(b)

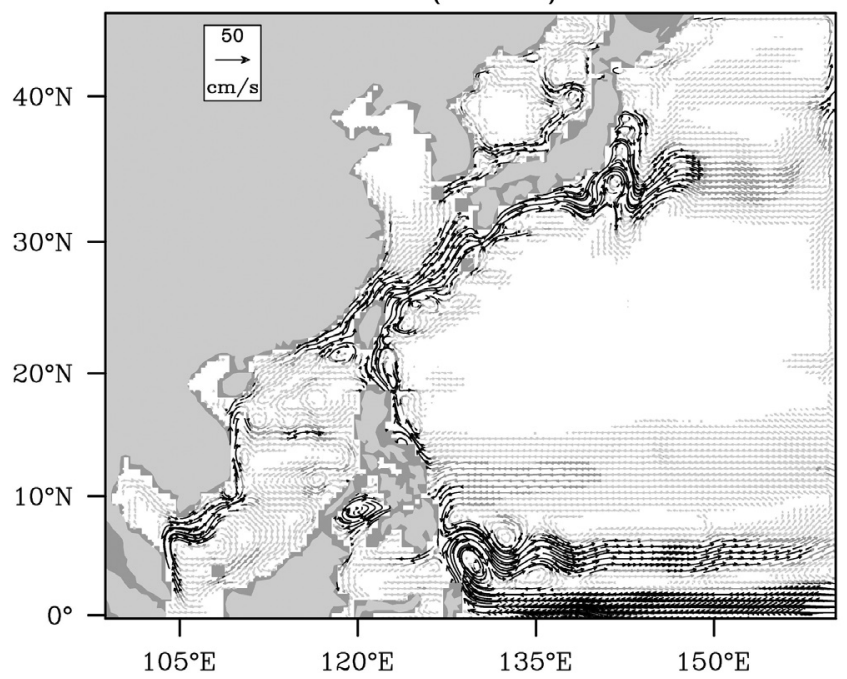

Fig. 5. Depth-averaged monthly mean surface velocity $\left(\mathrm{cm} \mathrm{s}^{-1}\right)$ in (a) January and (b) July derived from the year 10 of integration of the control experiment. The depth-averaging is over the top $50 \mathrm{~m}$ or entire water column for which is shallower. Only velocities greater than $5 \mathrm{~cm} \mathrm{~s}^{-1}$ are plotted. The black vector denotes velocity greater than $20 \mathrm{~cm} \mathrm{~s}^{-1}$, whereas the dark grey vector denotes velocity ranges between 15 and $20 \mathrm{~cm} \mathrm{~s}^{-1}$.

winter. This averaged bifurcation latitude, as well as the seasonal variation in the latitude, agrees with the climatological analysis of Qu and Lukas (2003). They indicated that such seasonal variation corresponded well with the local Ekman pumping associated with the Asian monsoon winds.

The northern branch of the NEC turns into the Kuroshio, which flows off the east coast of Taiwan and then turns northeastward following the southern coast of Japan. The Kuroshio intrusion into the SCS through the Luzon Strait exhibits large seasonality with a maximum occurring in winter. Similar seasonal variation in the Kuroshio intrusion has been revealed by several earlier studies made use of observation data (e.g., Qu 2000) or numerical model results (e.g., Xue et al. 2004). In winter, the intruded Kuroshio forms an anticyclonic current loop west of the Luzon Strait. The current loop bifurcates near the shelf break with the westward branch merges into the cyclonic circulation in the northern SCS. This cyclonic circulation vanishes when the Kuroshio intrusion decreases in summer. The currents in the Taiwan Strait are also reproduced well, with a maximum occurring in summer. To the south of the NEC, the NECC is weaker in winter and stronger in summer, while the NEC is stronger in winter. The SEC presents less seasonality in the control experiment.

Figure 6 shows the depth-averaged transports along various cross sections for all seven climatological experiments. All the experiments, except SP and noCndg, exhibit the aforesaid seasonality of the Kuroshio intrusion, and the transport through the Taiwan Strait. The poleward transport of the Kuroshio through the section along $20.5^{\circ} \mathrm{N}$ can also be indicative of the variation in the Kuroshio intrusion into the SCS. When there is a decrease in the poleward trans- port, an increase in the amount of the Kuroshio intrusion is also observed. The northward transports through the Taiwan Strait increase in early spring and reach a maximum at about 2.0 Sv in summer, then decrease apparently in fall and approach minimum value at about $0.9 \mathrm{~Sv}$. While these peak values of the simulated transports are slightly larger (smaller) in winter (summer) as compared with the observed values reported in Jan et al. (2006), the seasonal variations are reasonable. The transports across the section $25^{\circ} \mathrm{N}$ show relatively weak seasonal variations; such reduction of the seasonal variations is also revealed by the numerical model results of Kagimoto and Yamagata (1997). On the other hand, SP and noCndg produce larger transport through the 25 and $20.5^{\circ} \mathrm{N}$ sections as compared with the others, resulting in less transport through the Taiwan Strait. It should be recalled that both experiments exhibit a sharp decrease of kinetic energy in time. A further examination reveals that the SEC and NECC for the two experiments are significantly weaker than those in the control experiment in all seasons (Figures not shown). This could be a possible explanation for the decrease in the column-averaged kinetic energy.

The monthly mean SST obtained from the control experiment is given in Fig. 7. The spatial distribution of the SST is broadly in good agreement with the WOA98 climatology. This is probably due to the fact that the surface heat flux correction is used in the upper boundary condition for the control experiment. The difference between the winter and summer is apparently larger in the simulated SST. Larger variations also occur over the marginal seas in the model output. Overall, the warm tongue along the Kuroshio path is well simulated. The SST distribution over the SCS is also indicative of the seasonal variations in the near surface 
currents. The cyclonic circulation carries cold water southward along the coast of Vietnam in the winter, whereas the anticyclonic circulation brings the warm water northward in the summer. Lastly, the large SST gradient along part of the eastern boundary may be due to ill-specification of the lateral boundary conditions there.

Figure 8 presents the scatterplots of the monthly mean SST for the last year model integration output. The x-axis is the modeled SST, and y-axis is the SST from WOA98 monthly climatology. Each marker on the plot represents a single pixel which is defined by an area-average of each 10 $\times 10$ grid box in the model domain. The pixels are divided by three latitude bands as denoted in each panel. The control experiment has good correspondence with the WOA98 climatology. Generally, noCndg and noFC slightly underestimate $S S T$ to the north of $20^{\circ} \mathrm{N}$; noSP underestimates (overestimates) over the higher (lower) latitudes. Nevertheless, these model results are in general agreement with the observation. On the other hand, SP and Cndg show significant departures from the observation. SP underestimates SST in most of the latitudes, and Cndg overestimates SST, particularly in the high SST regions. A further investigation shows that a cold bias of SP occurs in all seasons, while a warm bias of Cndg mainly occurs in the warm season (April to September). The results presented here are consistent with those shown in Fig. 4c. We have also checked similar scatterplots for the heat contents (in the upper $400 \mathrm{~m}$ ) drawn from the experiments against those derived from WOA98 climatology. All the experiments, except SP and Cndg, exhibit a similar agreement with the observation (Figures not shown). SP and Cndg produce a similar underestimate and overestimate, respectively. However, the departures of the heat content for SP and Cndg are relatively moderate as compared with their SST deviations. The result implies that the vertical thermal structure in the upper ocean must have large differences among these experiments.

Three additional experiments are conducted to evaluate the sensitivity of the model to the nudging toward momentum climatology. The experiments adopted the settings for the control experiment, and the noSP and noFC, except that they all dropped the nudging term in the momentum tendency equation. The results exhibit an increase of simulated kinetic energy in all three cases (Figure not shown). However, very little changes are found in the temperature fields as compared with their corresponding original experiments. Therefore, they will not be further discussed. Last, because differences between the results from noFC and noQC are insignificant, only the former will be included in further discussion.

\subsection{Seasonality and Regionality}

The seasonal variations of the experiments are examined using the area-averaged sea surface temperature (SST), heat content ( $\mathrm{HC}$, in the upper $400 \mathrm{~m})$, and the isothermal depth (ID, as referred to by Qu et al. 2007). The isothermal depth is defined as the depth where the water temperature is $0.8^{\circ} \mathrm{C}$ cooler than the near-surface value at $10 \mathrm{~m}$. For calcu-
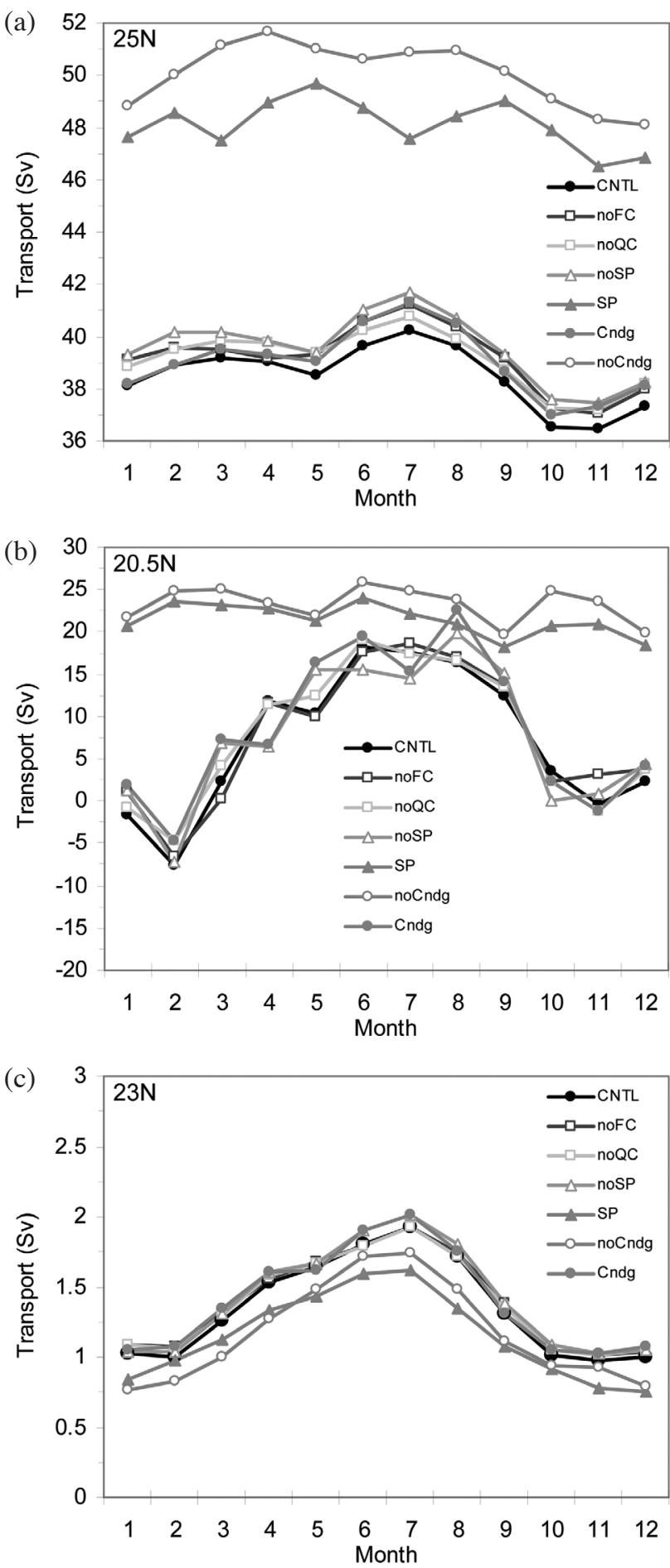

Fig. 6. Transport (Sv) from surface to bottom for the sections along (a) $25^{\circ} \mathrm{N}$, (b) $20.5^{\circ} \mathrm{N}$, and (c) $23^{\circ} \mathrm{N}$. The section along $25^{\circ} \mathrm{N}$ extends from 121.75 to $126.25^{\circ} \mathrm{E}$, the section along $20.5^{\circ} \mathrm{N}$ extends from 120.25 to $122.25^{\circ} \mathrm{E}$, and the section along $23^{\circ} \mathrm{N}$ across the Taiwan Straits. The transports are derived from the year 10 of integration. 

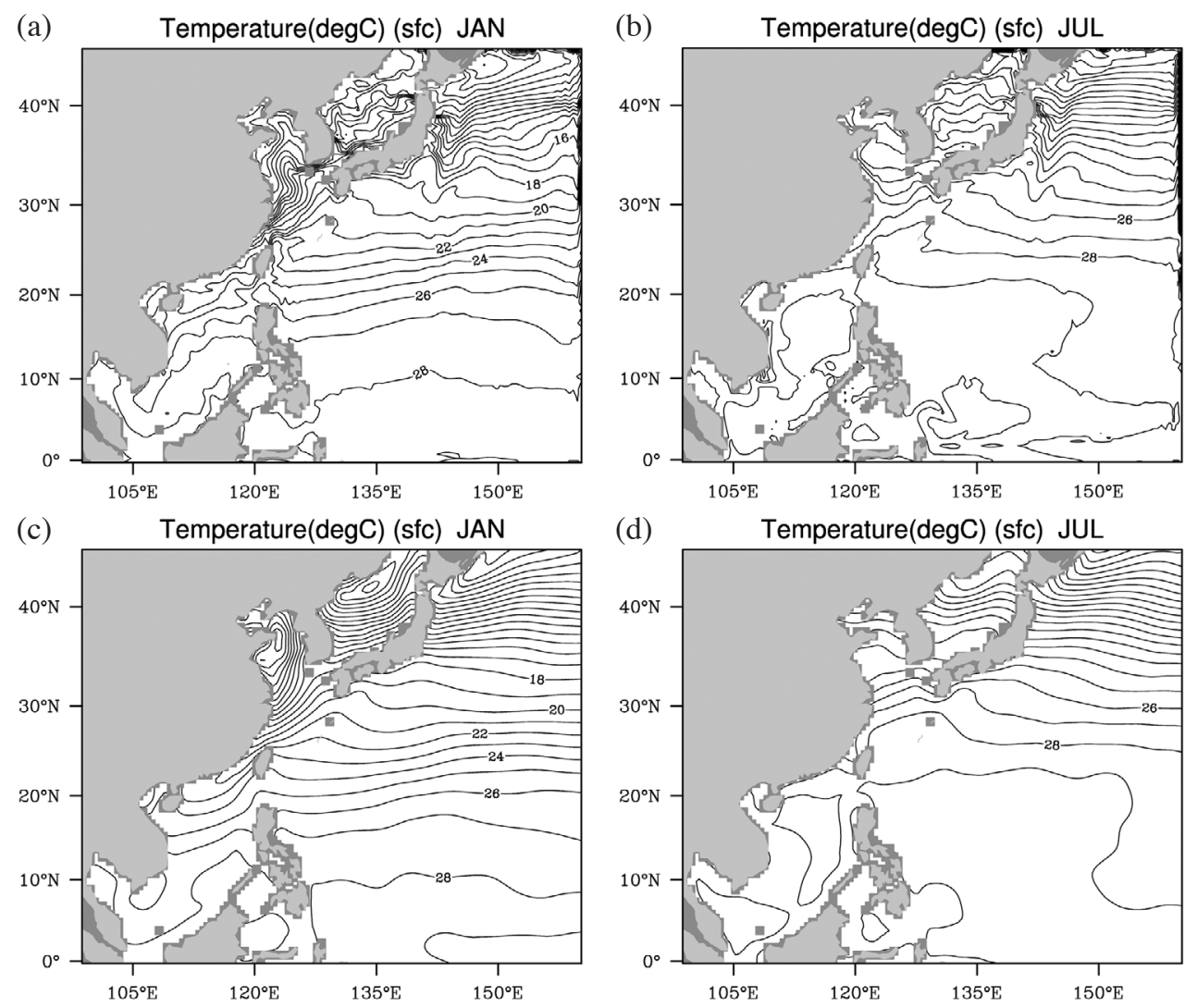

Fig. 7. Sea surface temperature (in Celsius) in (a) January and (b) July derived from the year 10 of integration of the control experiment, and in (c) January and (d) July derived from the WOA98.
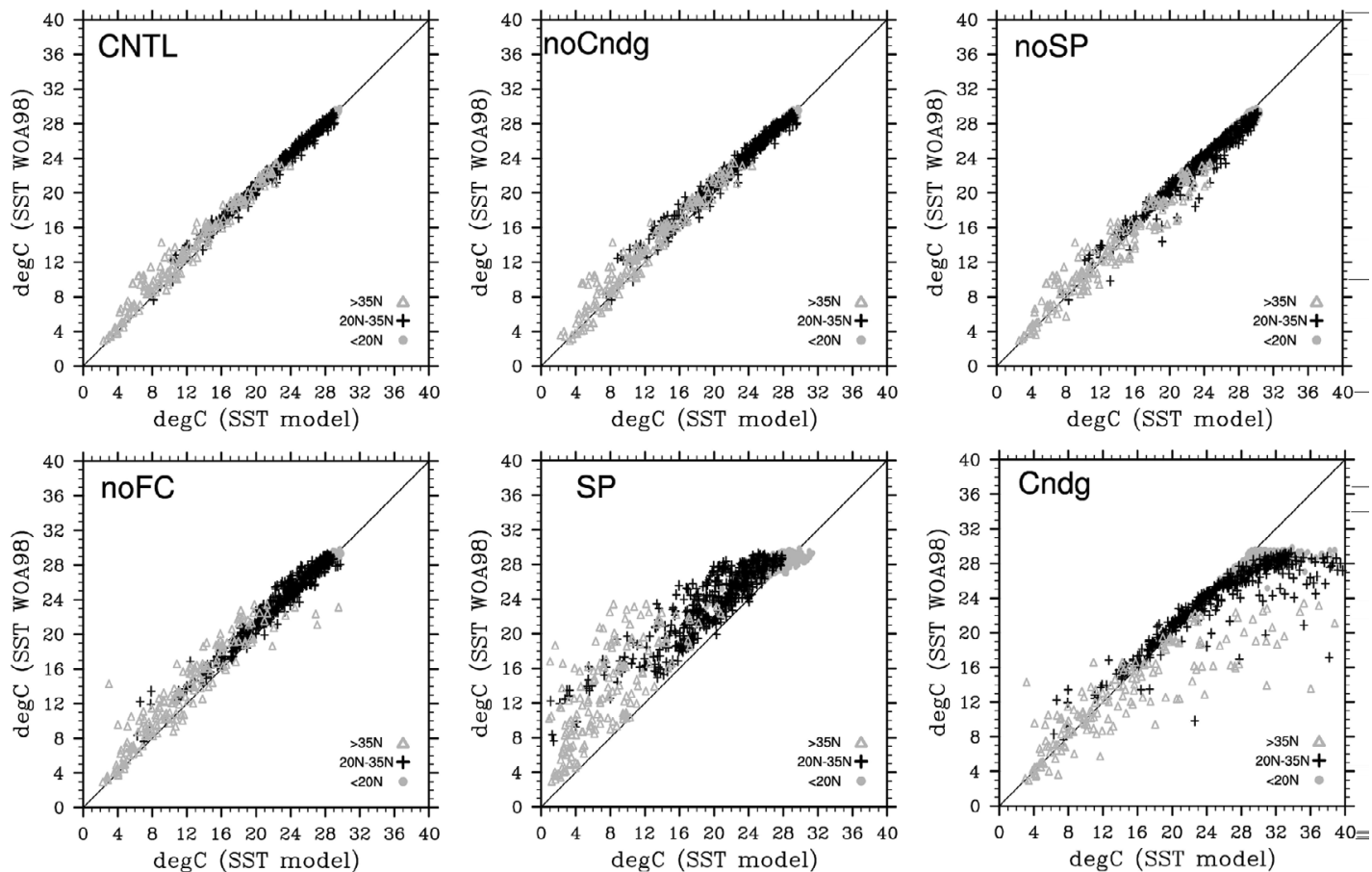

Fig. 8. Scatterplots of monthly mean SST (in Celsius) for experiments CNTL, noCndg, noSP, noFC, SP, and Cndg. The abscissa is the modeled SST, and the ordinate is the SST from WOA98 monthly climatology. The black solid diagonal line is a $1: 1$ line. The filled circle, cross sign, and hollow triangle represent the pixels in latitudes to the south of $20^{\circ} \mathrm{N}$, between $20-35^{\circ} \mathrm{N}$, and to the north of $35^{\circ} \mathrm{N}$, respectively. The value for an individual pixel is an area-averaged of each $10 \times 10$ grid box in the model domain. The model outputs are taken from the year 10 of the integration. 
lating the isothermal depth, the temperature profile at each model grid point was first interpolated from the sigma grid to a specific $z$ level grid. The isothermal depth is a good proxy for mixed layer depth. Thus it is denoted as mixed layer depth (MLD) hereafter.

Table 2 presents the seasonal ranges and the annual mean of SST, HC and MLD for each region. The values are derived from WOA98 monthly climatology. The annually averaged SST and HC decrease from the south to the north, as seen from SCS to ETW, and to SJP. The HC of ECS is much smaller than the others, mainly due to its shallowness. The shallowest MLD appears in SCS and the deepest MLD occurs in SJP. On the other hand, the largest seasonal range of $S S T$ is found in $E C S$, while the largest seasonal range of HC and MLD are found in SJP. The SCS exhibits the smallest seasonal ranges for SST, HC and MLD. The results indicate a geographical dependence of HC and MLD in these regions. The HC not only depends on the SST, but also depends on the temperature profile. Because the temperature profile is an important parameter in determining the MLD, both the SST and MLD are critical to reasonable simulation of $\mathrm{HC}$.

Figure 9 shows the time series of area-averaged SST,
HC and MLD. The variables derived from the WOA98 monthly climatology are also imposed. Climatologically, there is distinct seasonality for SST, HC and MLD in all regions. The SST and HC present higher (lower) values in warm (cold) season. On the other hand, the MLD exhibits shallowest (deepest) depths in warm (cold) season. The months from April to September are denoted as the warm season, and the rest are referred to as the cold season, hereafter.

In general, the simulated SST and HC follow the climatology well in terms of the seasonal variations for all the experiments in all regions. The departures from the climatology, mostly revealed by SP, noCndg, and Cndg, are similar in all regions. Significant warm biases of SST are found in the warm season for experiment $\mathrm{Cndg}$, with the largest value of about $48^{\circ} \mathrm{C}$ appearing in ECS. Despite that significant SST warm biases are produced in the warm season for all the regions, the departures of $\mathrm{HC}$ from the climatology are relatively small. There are distinct long-term drift of $\mathrm{HC}$ for SP and noCndg, while significant SST drift only occurs for SP.

Table 3 gives the departures of annual mean SST and $\mathrm{HC}$ for year 10 of the integration. It can be seen that lower

Table 2. The seasonal range and annual mean of sea surface temperature, heat content and mixed layer depth (MLD, also the isothermal depth) for the four averaged regions. The variables are derived from the WOA98 monthly climatology. The seasonal range is calculated as the difference between the maximum and minimum values.

\begin{tabular}{|c|c|c|c|c|c|c|}
\hline & \multicolumn{3}{|c|}{ Seasonal Range } & \multicolumn{3}{|c|}{ Annual Mean } \\
\hline & $\Delta \mathrm{SST}$ & $\Delta \mathrm{HC}$ & $\Delta$ MLD & SST & $\mathrm{HC}$ & MLD \\
\hline ECS & 11.2 & 1.9 & -91.1 & 21.9 & 5.4 & -47.2 \\
\hline ETW & 6.4 & 2.0 & -97.3 & 25.6 & 33.4 & -55.4 \\
\hline SCS & 3.5 & 1.3 & -32.0 & 27.6 & 27.9 & -33.5 \\
\hline SJP & 8.7 & 2.7 & -150.7 & 23.0 & 30.4 & -61.9 \\
\hline
\end{tabular}

Table 3. The departures of annual mean sea surface temperature and heat content. The units are ${ }^{\circ} \mathrm{C}$ and $10^{9} \mathrm{~J}^{-2}$, respectively. The departure is defined as the difference between year 10 of the integration and the WOA98 climatology.

\begin{tabular}{|c|c|c|c|c|c|c|c|c|c|c|c|c|}
\hline & \multicolumn{6}{|c|}{ SST } & \multicolumn{6}{|c|}{$\mathrm{HC}$} \\
\hline & CNTL & noFC & noSP & noCndg & SP & Cndg & CNTL & noFC & noSP & noCndg & SP & Cndg \\
\hline ECS & -0.59 & -1.08 & 0.14 & -1.19 & -4.94 & 5.47 & -0.13 & -0.16 & -0.12 & -0.58 & -1.27 & 0.49 \\
\hline ETW & -0.40 & -0.62 & 0.09 & -0.75 & -1.09 & 1.85 & -5.56 & -5.60 & -5.69 & -6.66 & -7.32 & -5.48 \\
\hline SCS & -0.10 & -0.24 & 0.40 & -0.24 & -2.07 & 2.05 & -0.03 & -0.07 & -0.15 & -1.13 & -1.79 & 0.05 \\
\hline SJP & -0.58 & -1.11 & 0.02 & -0.71 & -2.92 & 1.13 & -0.43 & -0.53 & -0.50 & -1.84 & -3.46 & -0.38 \\
\hline
\end{tabular}


(a)

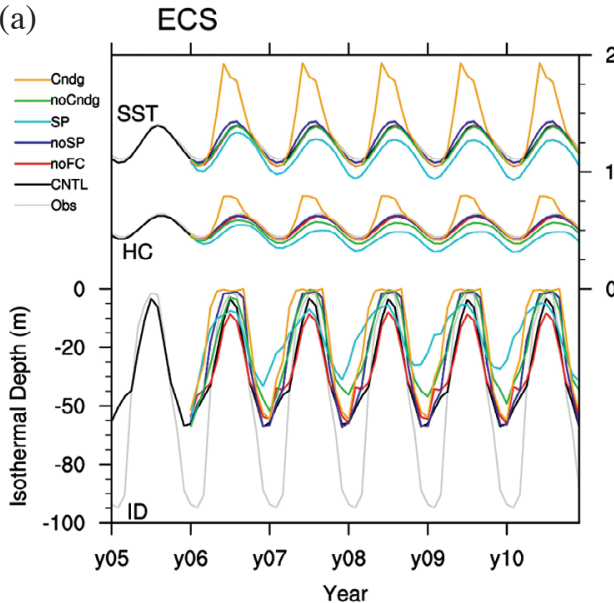

(c)
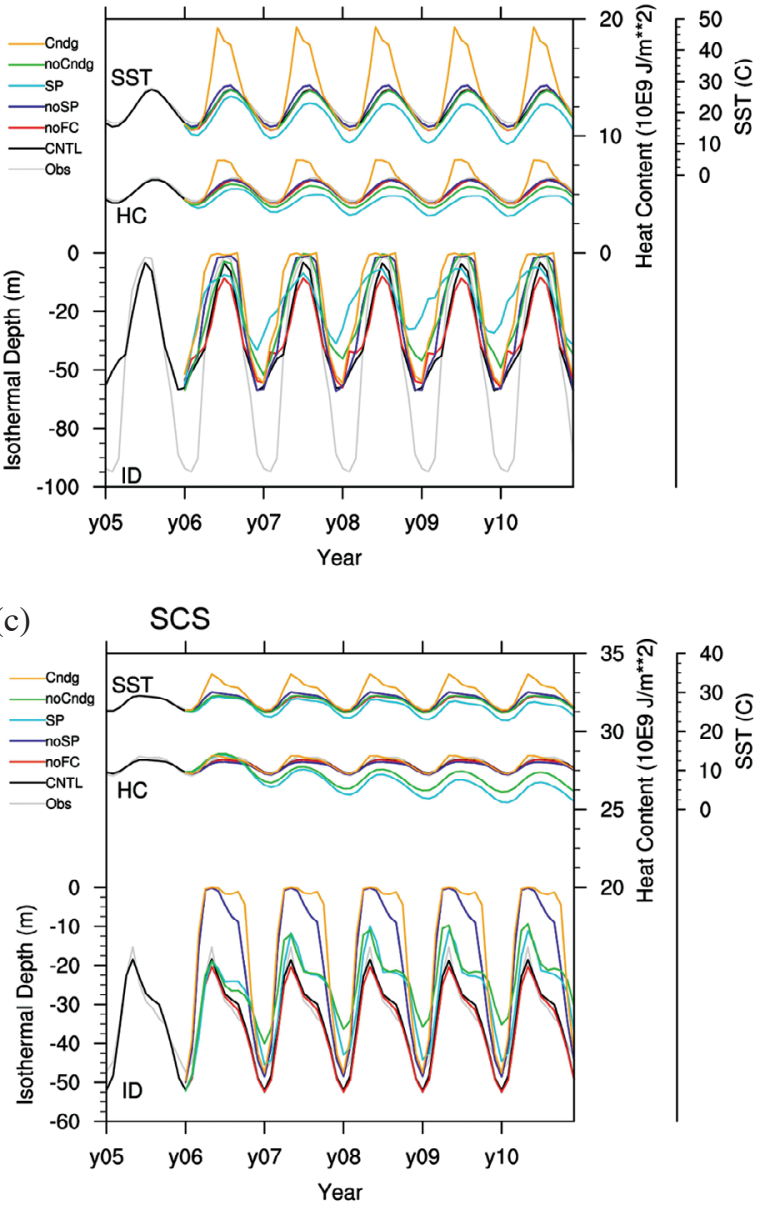

(b)

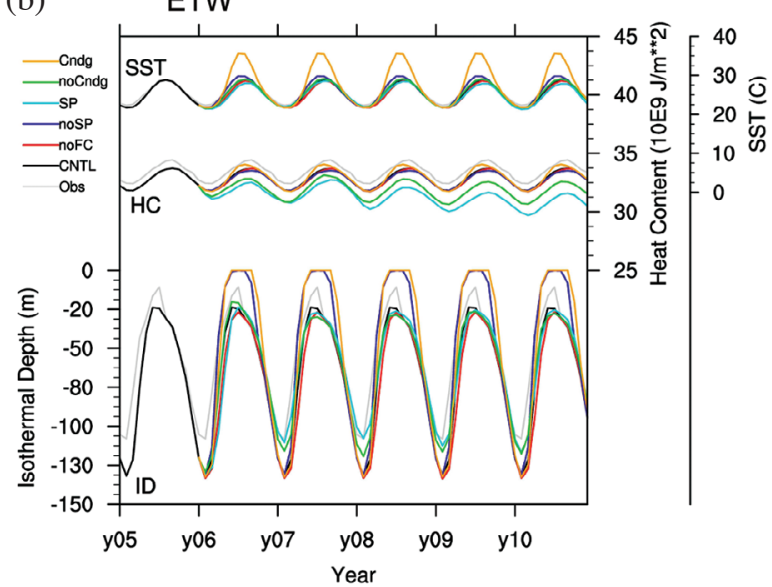

(d)

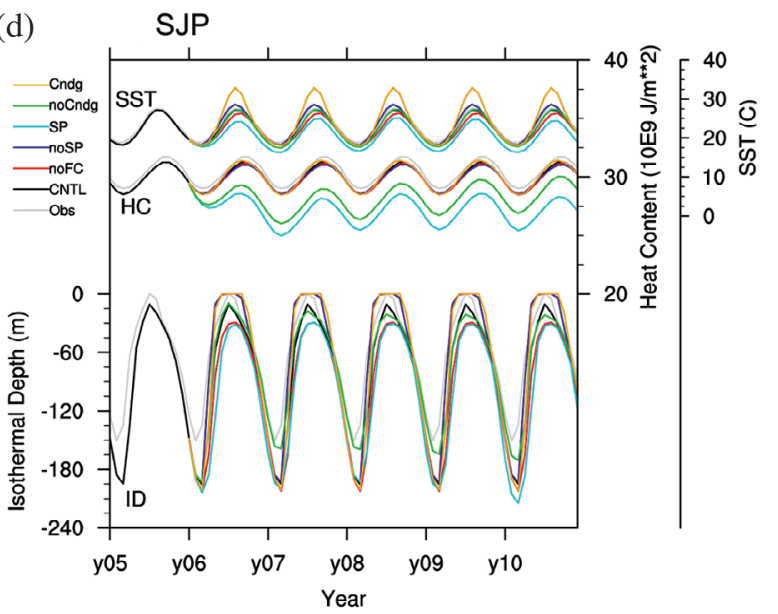

Fig. 9. Time series of area-averaged SST (in Celsius), heat content $\left(\mathrm{J} \mathrm{m}^{-2}\right)$, and isothermal depth (m) for regions (a) ECS, (b) ETW, (c) SCS, and (d) SJP. The grey lines are derived from the WOA98 monthly climatology for each variable over the same region. The model outputs (monthly mean, in different colors as denoted on the plots) are taken from year 5 to year 10 of the integration.

SST are found in all regions for experiment SP, corresponding to larger decreases of HC. However, the lowest SST does not necessarily correspond to the largest decrease in HC. Similar results are found for noCndg, though the changes are more modest. Both Cndg and noSP overestimate SST in all regions, whereas only Cndg slightly overestimates $\mathrm{HC}$ in ECS and SCS. The result confirms the aforementioned notion that the $\mathrm{HC}$ departures are out of proportion with respect to the SST warm biases for Cndg.

There is large diversity of differences in MLD among all experiments, especially in the cold season. In the warm season, noSP and Cndg produce shallowest MLD in all regions. Actually the MLD predicted by the two experiments almost vanishes for a few months in the warm season. The performances of other experiments do not show consistency among different regions, but all produce considerable MLD in the warm season. In the cold season, the MLDs are underestimated by all the experiments in ECS. Generally, CNTL and noFC produce deeper MLD than noCndg and SP. The effects of different terms applied to these experiments on the MLD in cold season are still unclear.

The area-averaged temperature profiles for January and July are shown in Fig. 10. There are significant departures for SP and noCndg in all regions, particular in ECS. The characteristics of the departures for the two experiments are similar in the four regions. SP exhibits larger cold biases in the upper layers, while noCndg shows larger cold biases in the deeper layers. As for the other experiments, the temperature profiles are reasonably close to the WOA98 climatology. In the summer, similar features are found in SP and noCndg. Despite the cold biases, SP maintains a similar vertical structure as the WOA98 climatology. Cndg and noSP predict stably stratified water column in the top $20 \mathrm{~m}$ in all regions, due to the intense warm biases in the simulated SST. The warm bias in Cndg is much stronger than that in noSP. As a result of the intense warm biases, the shapes of the temperature profiles are apparently different from the WOA98 climatology. The structural difference of Cndg can be used to explain the seemingly inadequately small $\mathrm{HC}$ departures corresponding to the very large $S S T$ warm biases. 
(a)

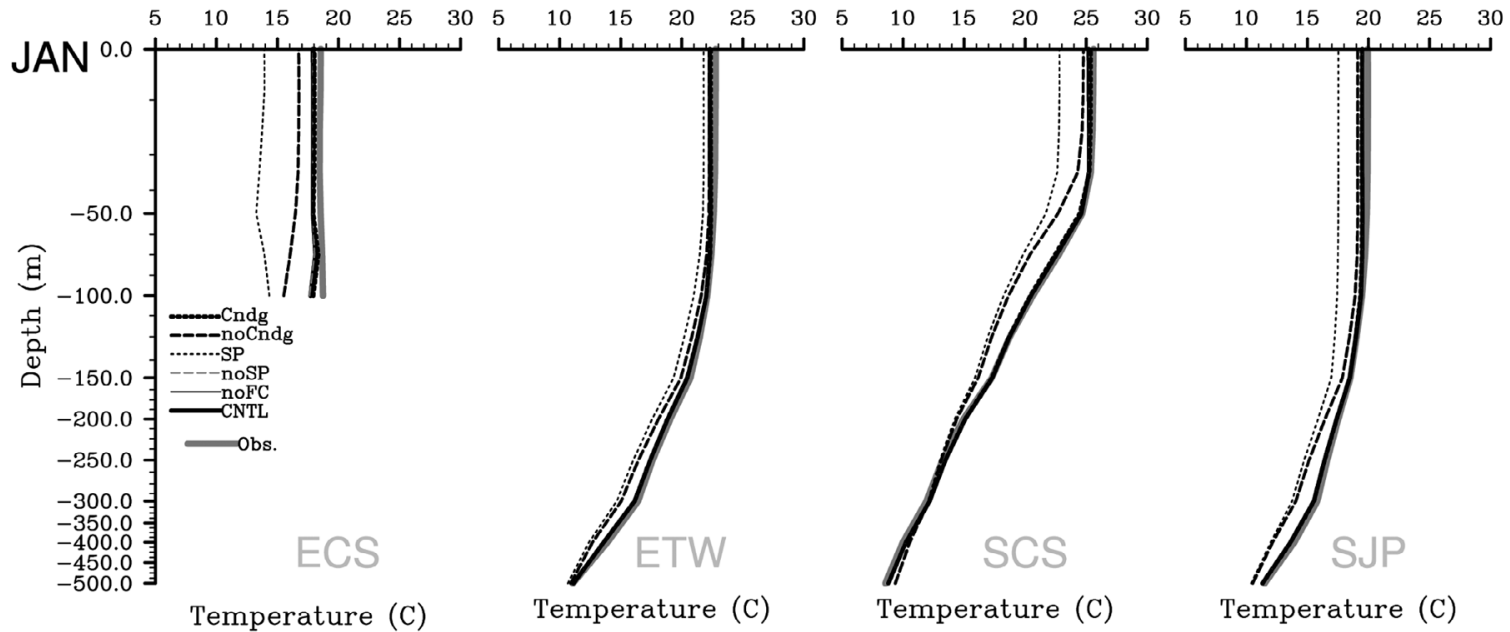

(b)

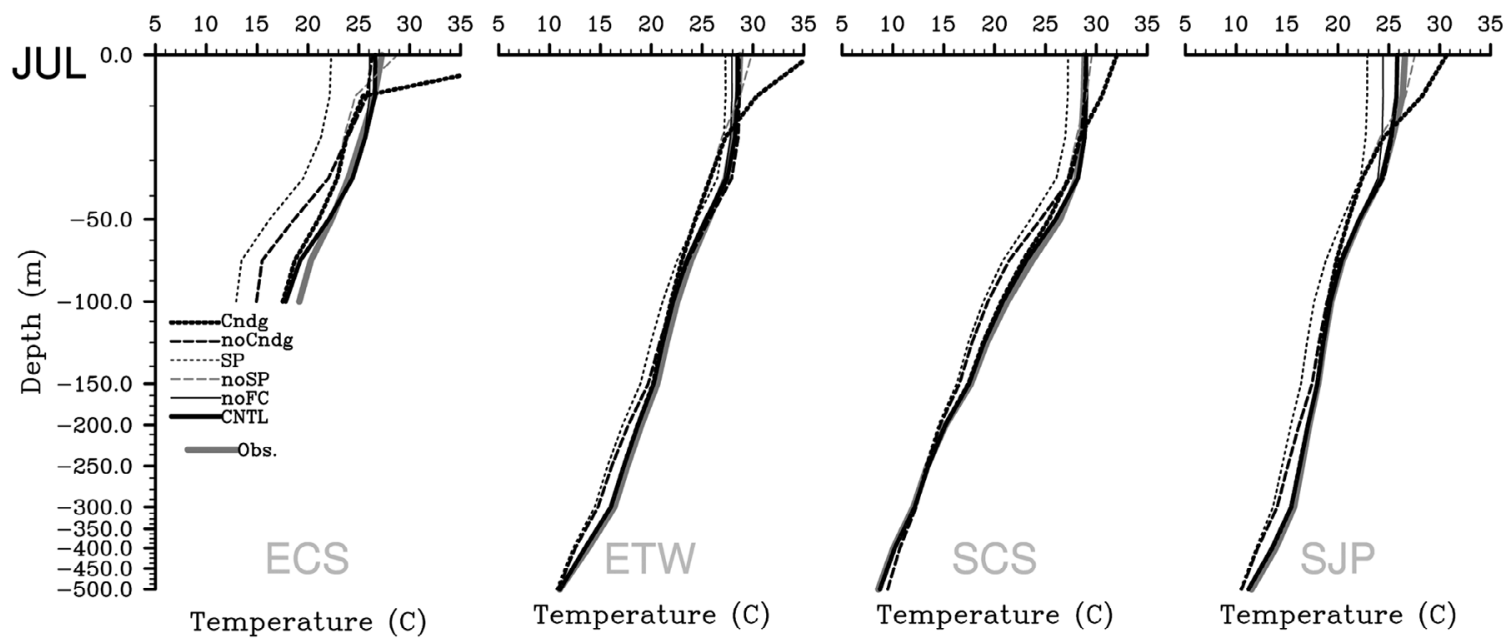

Fig. 10. Area-averaged (a) January and (b) July temperature (in Celsius) in regions ECS, ETW, SCS, and SJP derived from WOA98 monthly climatology (grey solid thick line) and selected experiments (as denoted on each panel). The model outputs are taken from the year 10 of the integration.

The aforesaid seasonal relationship between the $\mathrm{HC}$ and $S S T$, as well as the differences among the experiments, can be summarized in Fig. 11. The close correspondence between the seasonality of the $\mathrm{HC}$ and $S S T$ is evident in all cases, as revealed in Fig. 9. CNTL and noFC is apparently better than the others as compared with the WOA98 climatology. The simulated SSTs of the two experiments are quite good in all regions, while the amounts of the simulated HC are smaller than the WOA98 climatology. Cndg and noSP overestimate the SST in the warm season for all regions, while only Cndg overestimate the $\mathrm{HC}$ in ECS. It is recalled that no solar penetration is applied for the two experiments, namely Cndg and noSP, and no flux corrections are applied to Cndg. The absence of the solar penetration in the two experiments results in an unrealistic upper ocean temperature profiles in summertime, as can be seen in Fig. 10b. The less intense SST warm bias of noSP is mainly due to the flux correction, and will be addressed later. Both SP and noCndg underestimate the HC significantly, while only SP underestimates the SST for all seasons. Both experiments adopt the solar penetration and omit the nudging term in the five years integration. The absence of the nudging term tends to result in a much smaller heat storage capacity in the upper ocean than the others. The flux correction in noCndg acts to increase the upper ocean heat storage by adjusting the SST toward the WOA98 climatology.

The results herein indicate that solar penetration is important for development and maintenance of a reasonable upper ocean thermal structure. However, apparent cold biases are found when solely the solar penetration is used. The flux correction tends to adjust the near surface temperature, while the nudging term is able to nudge the temperature profile toward the climatology. It should be stressed that unrealistic temperature profile is produced when the nudging term is applied without flux corrections. However, a combination of solar penetration and the nudging term appears adequate 
(a) ECS

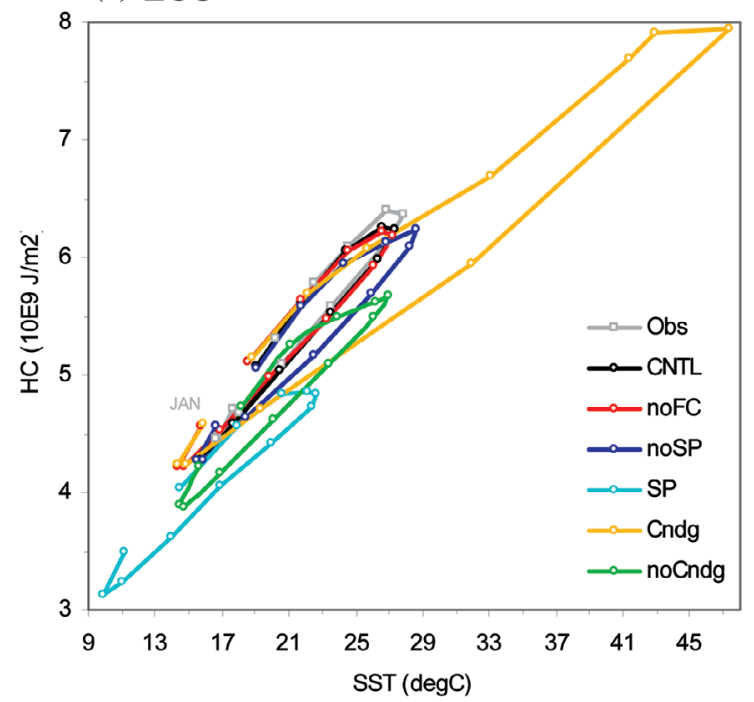

(c) SCS

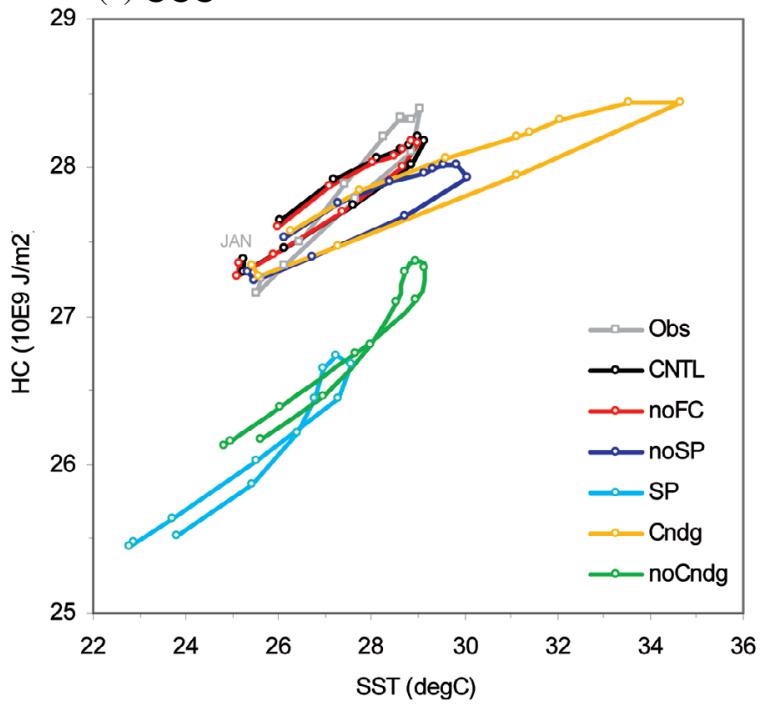

(b) ETW

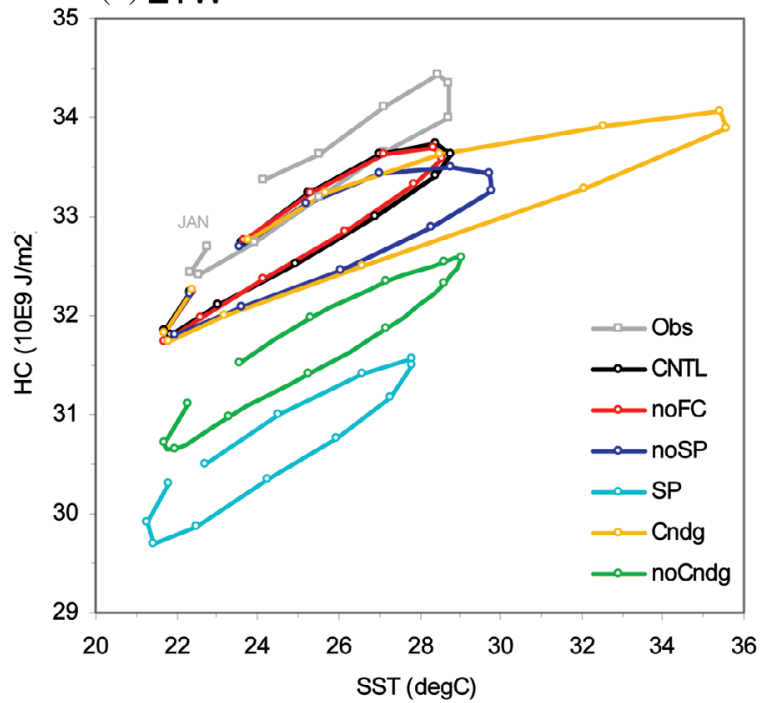

(d) SJP

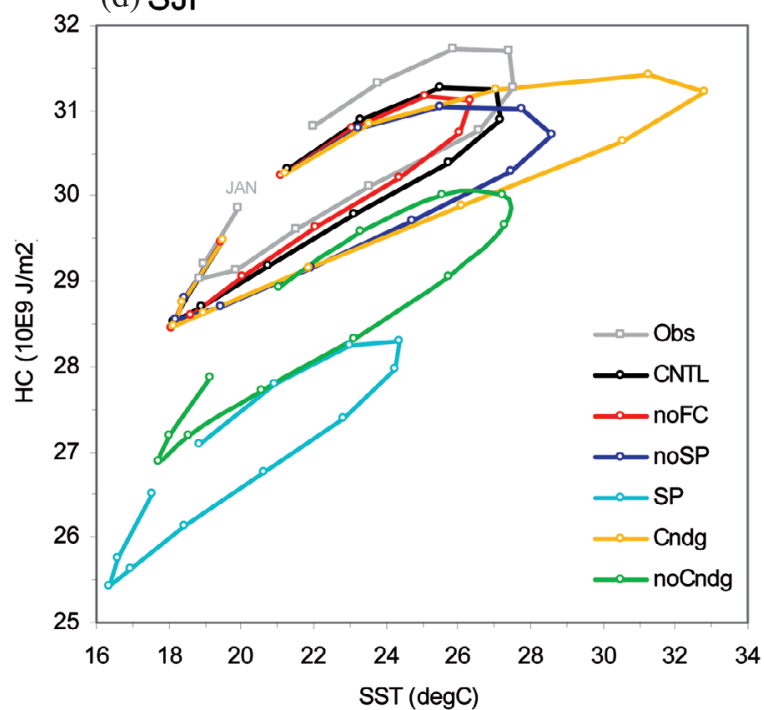

Fig. 11. Heat content $\left(\mathrm{J} \mathrm{m}^{-2}\right)$ as a function of sea surface temperature (in Celsius) averaged over the regions (a) ECS, (b) ETW, (c) $S C S$, and (d) $S J P$. The abscissa is the heat content and the ordinate is the SST. The variables are monthly mean values derived from the WOA98 monthly climatology (grey) and selected experiments (as denoted in each panel). The heat content is integrated in the upper $400 \mathrm{~m}$. The term "JAN" on each panel represents the month of January, indicating the start of the annual cycle for the observation. The model outputs are taken from the year 10 of the integration.

for simulation of upper ocean thermal structure. Hence, the flux correction is not necessary in this case.

\subsection{Flux Correction}

The extent of the adjustment for the net surface heat flux will be addressed herein. The net surface heat flux as a function of SST is presented in Fig. 12. For the control experiment, the adjustment is relatively smaller in the warm season. In the cold season, the net surface heat flux is adjusted to be less cooling when the simulated SST is underestimated. Similar adjustment appears in all regions. The adjustment in noCndg is similar as in the control experiment.
In noSP, the net surface heat flux has been intensely adjusted to produce less warming in the warm season. Despite the warm bias in the modeled SST in the warm season, the adjusted net surface heat flux is approaching zero.

Because net surface heat flux includes the solar radiation, a near zero net surface heat flux in the warm season implies an artificially imposed heat loss at the sea surface. Ezer (2000) attributed the deficit in his simulated heat content of the upper layers to the uncertainties in the parameterization of turbulence in the model, and the underestimate of the observed surface heat flux. A heat flux correction is applied in his study to mainly compensate for the errors in the observed surface heat flux. However, the flux correc- 
tion term shall compensate for errors in both the observed surface heat flux and the ocean model itself. According to Eq. (2), larger flux adjustment may occur, if the SST differs significantly from the reference temperature. A large correction coefficient represents a case where the model heat flux is dominated by the observed sea surface temperature rather than by the observed surface heat flux. The primary weakness in formulating the net surface heat flux with a correction term is an insufficient knowledge of the appropriate value of the correction coefficient to be used.

\subsection{Vertical Mixing}

The development of the oceanic boundary layer depends on the surface forcing and on the oceanic buoyancy and velocity profiles. To characterize the extent of the oceanic boundary layer, Large et al. (1994) defined a bulk Richardson number relative to the surface as:

(a) ECS

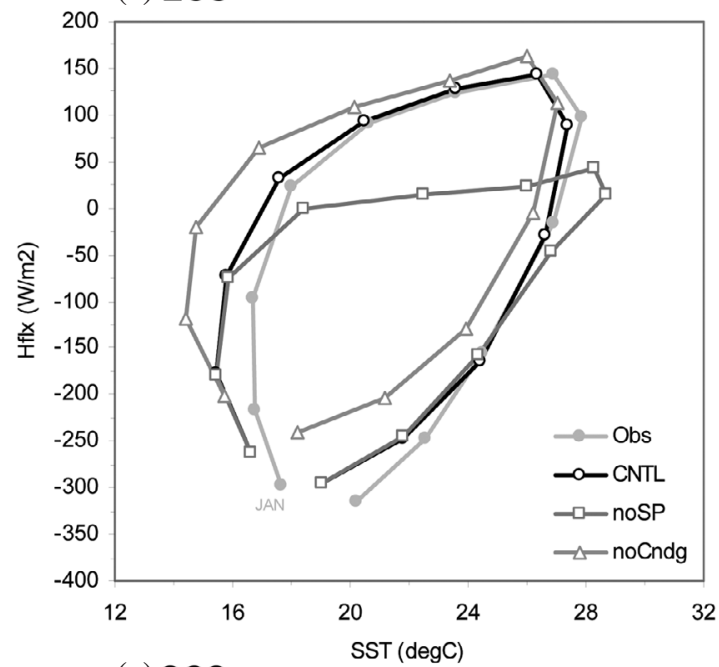

(c) SCS

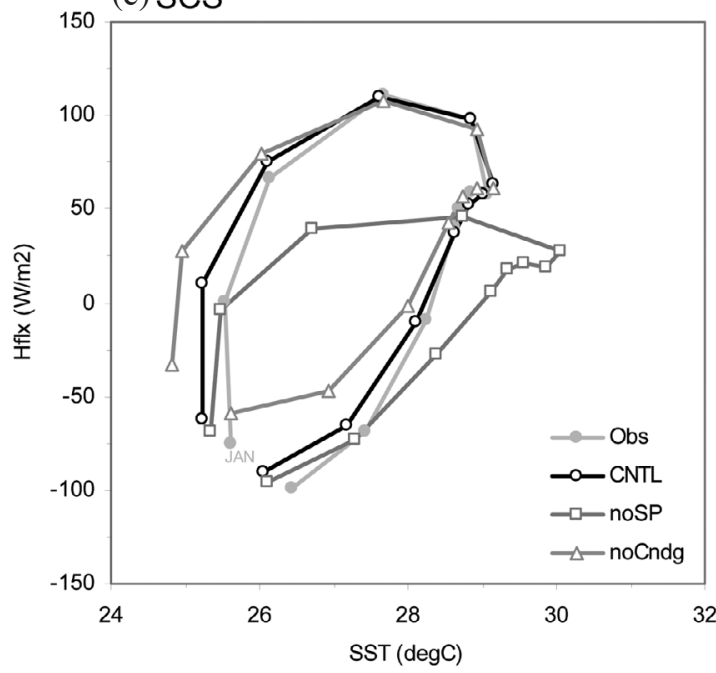

$\operatorname{Ri}_{b}(d)=\frac{\left[B_{r}-B(d)\right] d}{\left|V_{r}-V(d)\right|^{2}+V_{t}(d)}$

When the bulk Richardson number $\operatorname{Ri}_{b}(d)$, which is a function of depth, equal to a critical value, the depth $(d)$ is called surface boundary layer depth (SBLD). The boundary layer eddies with mean velocity $V_{r}$ and mean buoyancy $B_{r}$ should be able to penetrate a depth, namely the SBLD, where they first become stable relative to the local buoyancy $B(d)$ and velocity $V(d)$. The $V_{t}(d)$ term is related to turbulent velocity shear. Furthermore, at all depths inside the SBLD, the magnitude of the mixing coefficients (viscosity and diffusivity) depends upon both the SBLD and the surface forcing. Accordingly, deeper boundary layers should contain larger and more efficient turbulent eddies. The detail of the KPP scheme is referred to Large et al. (1994).

Based on the previous analysis, the different experiments in the study result in a variety of the mixed layer

(b) ETW

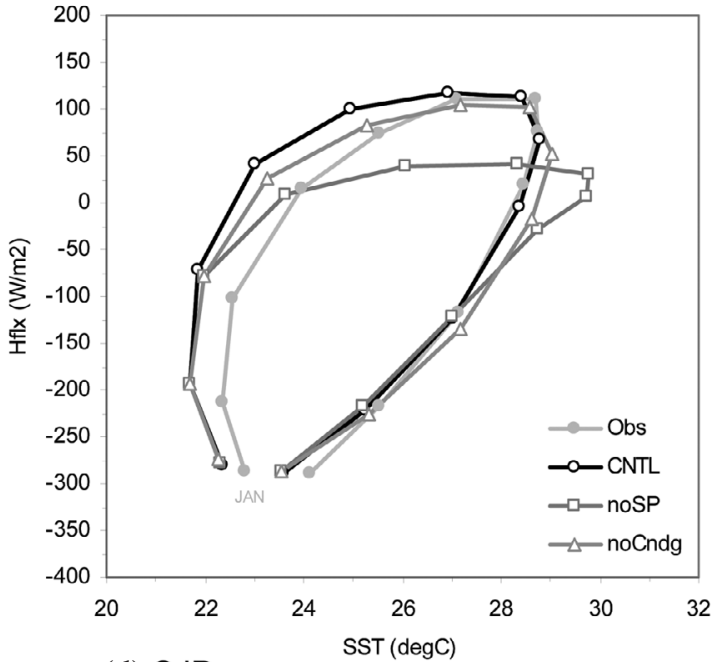

(d) SJP

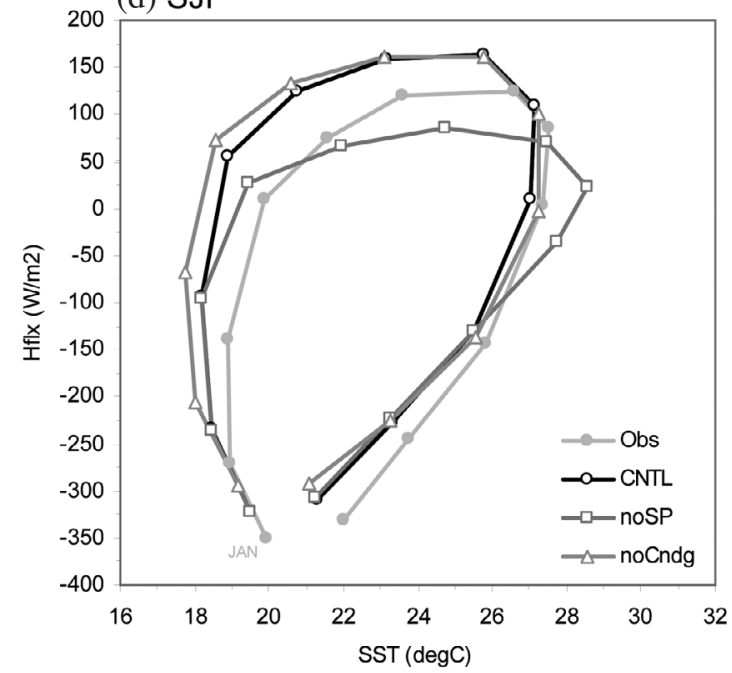

Fig. 12. Same as Fig. 11, but for the net surface heat flux ( $\mathrm{W} \mathrm{m}^{-2}$, including shortwave radiation) as a function of sea surface temperature (in Celsius). Only those experiments with flux correction are shown. 
depths. The evolution and maintenance of the mixed layer dynamics is still a topic for active research. Briefly, the development of mixed layer depth depends on the surface wind stress, and also depends on the stability of the underlying water and on the heat and freshwater balance through the surface. In the section, we interpret the differences among the experiments in terms of the SBLD and diffusivity $\left(K_{H}\right)$.

Figure 13 shows the area-averaged vertical temperature diffusivity as a function of depth and time for the last year (year 10) of integration. The abscissa starts from January. The averaged region is ECS. The surface boundary layer depth (SBLD), the MLDs (also the isothermal depth) derived from model output and the WOA98 monthly climatology are also plotted. The distribution of diffusivity and SBLD, as well as the MLD, for noFC and the control experiment are similar as SP. Thus only noCndg, noSP, SP, and Cndg are shown. In Fig. 13, the upper and lower panels are experiments with and without the flux correction. While the left and right panels are experiments without nudging and without solar penetration, respectively.

There are several similarities for all experiments. A general description is given below. Both the SBLD and diffusivity show strong seasonal dependency. In the cold season, the diffusivity is larger and mixing can penetrate much greater depth resulting in deeper SBLD. The peak values of the diffusivity appear within the surface boundary layer. Below the SBLD, the diffusivity decreases significantly. In the warm season, the SBLD diminishes apparently. The diffusivity becomes extremely small in all depths as compared to that in the cold season. The seasonal variations of the MLD are similar to that of SBLD.

In addition to the aforesaid similarities, there are some differences among these experiments. The SBLDs in noSP and Cndg vanish in the warm season. It is recalled that there is no solar penetration in the two experiments. The temperature profiles of the two experiments reveal that the near
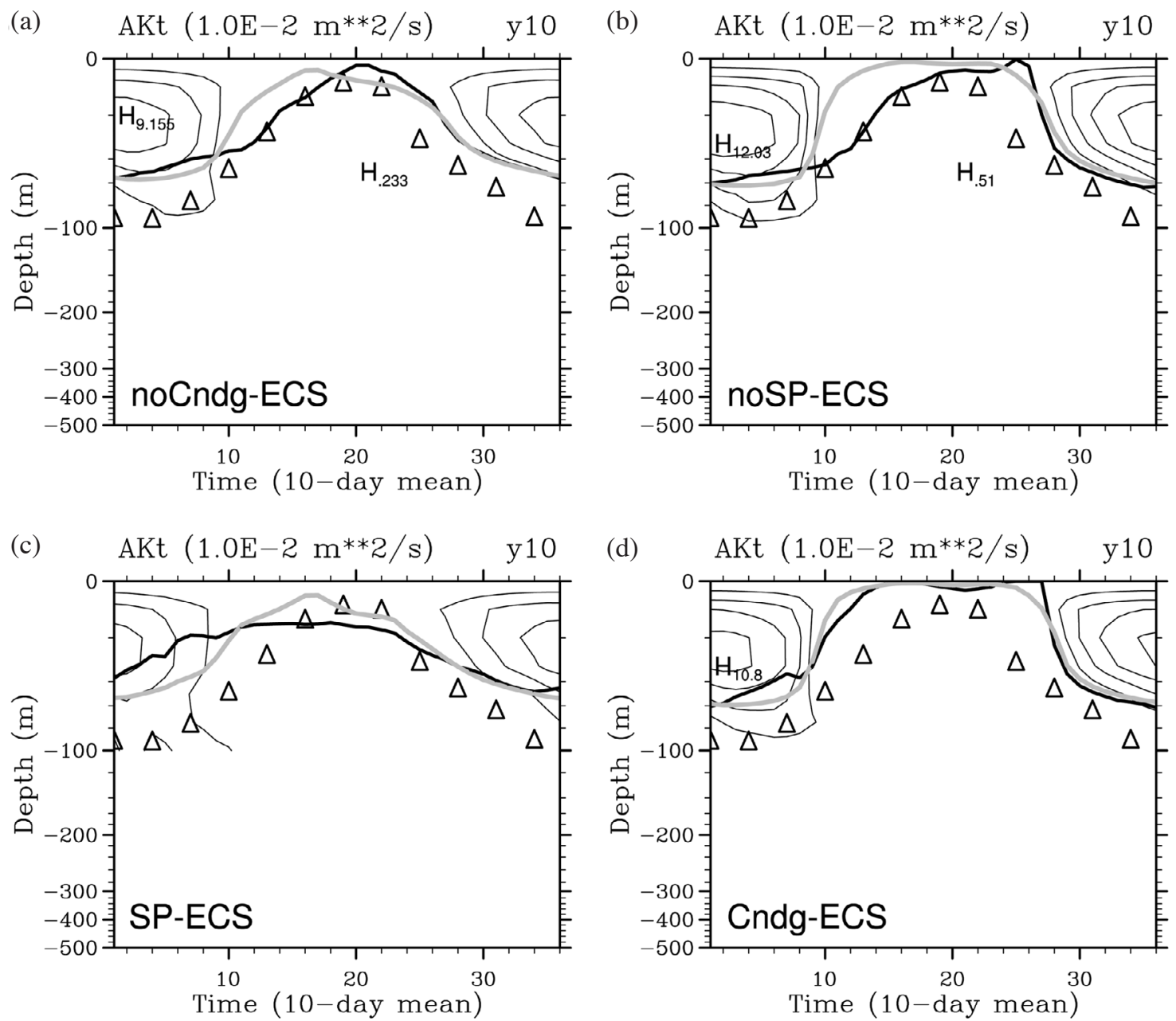

Fig. 13. Area-averaged vertical temperature diffusivity $\left(\mathrm{m}^{2} \mathrm{~s}^{-1}\right.$, contour) as a function of depth and time for experiments (a) noCndg, (b) noSP, (c) SP, and (d) Cndg for the last year (year 10 of integration). The averaged region is ECS. The boundary layer depth (in meters, grey lines) and isothermal depth (in meters, black lines) derived from model outputs are also imposed. The vertical temperature diffusivity and boundary layer depth are calculated from the KPP scheme. All these model derived variables are 10-day mean values. The hollow triangles denote the isothermal depth derived from the WOA98 monthly climatology. 
surface layer is very stable in summer, preventing effective vertical mixing (Fig. 10b). The result indicates that the inclusion of the solar penetration is a major factor for maintaining a summer SBLD, and mixed layer depth as well, as seen in noCndg and SP.

The situation is different in the cold season. The experiments with solar penetration (noCndg and SP) produce shallower MLD as compared with the experiments without solar penetration (noSP and Cndg). Despite very small differences found in the SBLD among these experiments, the larger peak values of diffusivities in noSP and Cndg imply stronger turbulent mixing in the cold season. This can also be seen in the temperature profiles from Fig. 10a. Furthermore, the experiments with and without a flux correction also present slight differences in the mixed layer depth during the cold season (compare the upper panels to the lower panels). In the winter, noCndg produces higher near surface temperature than SP; this is due to the adjustment of the flux correction (Fig. 10a). The well mixed layer is also deeper than that in SP, indicating a larger/deeper turbulent mixing in the upper ocean.

There are only slight structural differences in the temperature profiles between experiments with and without the flux correction in the other regions (Fig. 10). Thus very small differences are found in the diffusivity and SBLD in other regions. Figure 14 shows only the results for SP and
Cndg. Similar results are found as in region ECS: (1) Strong seasonal dependence of diffusivity and SBLD. (2) The solar penetration is a major factor for maintaining a mixed layer depth in the warm season. (3) The nudging term allows a deeper mixed layer depth in the cold season. In these regions with depths deeper than ECS, the MLD is deeper than SBLD especially in the cold season when the wind stirring is stronger and the turbulent mixing is very intense within SBLD. This is probably due to the Ekman pumping phenomena which is a nonlocal process in the mixed layer dynamics. Lastly, there are differences among the three regions. The seasonality, as well as the variation of MLD, in the SCS is smaller than others. This is partly due to the fact that the magnitude of the surface momentum fluxes is less variable in different seasons in the SCS.

During the warm season, there is a net surface heat flux from the atmosphere to the ocean (Fig. 3a). For the experiments without the solar penetration, the net surface heat flux causes intense warming at the sea surface when all the heat flux is applied as surface heating. Vertical turbulent mixing is suppressed in the stratified upper layers. Thus a diminutively shallow MLD is formed. When the summertime MLD is too shallow, the upper ocean temperature gradients are too large, and there is insufficient vertical mixing to transfer heat down from the surface layer. As a result, the temperature below the near surface layer (in about the top $20 \mathrm{~m}$ ) is

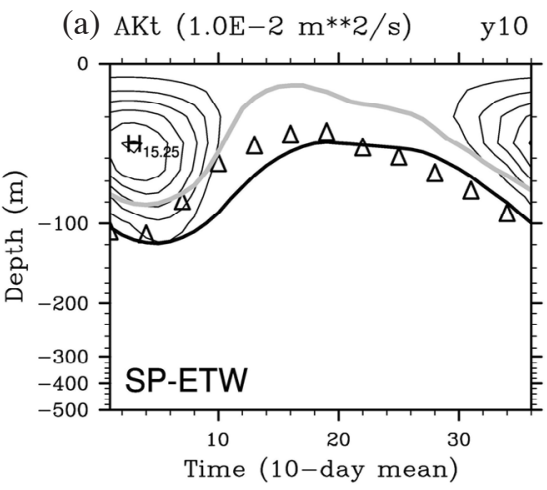

(d) AKt $(1.0 \mathrm{E}-2 \mathrm{~m} * * 2 / \mathrm{s}) \quad \mathrm{y} 10$

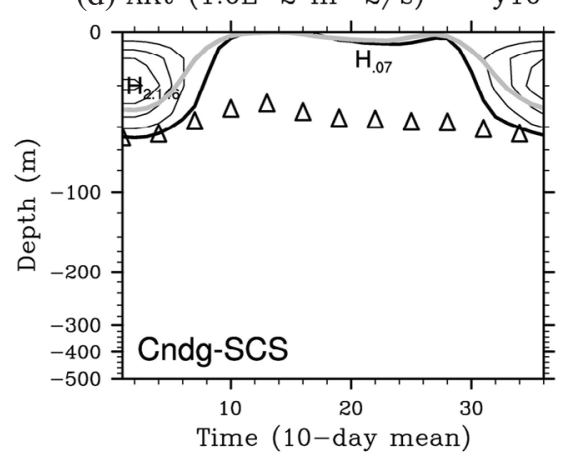

(b) AKt $(1.0 \mathrm{E}-2 \mathrm{~m} * * 2 / \mathrm{s}) \quad \mathrm{y} 10$

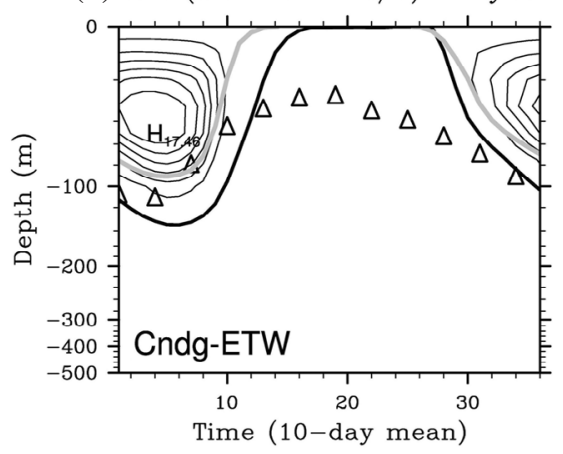

(e) AKt $(1.0 \mathrm{E}-2 \mathrm{~m} * * 2 / \mathrm{s})$

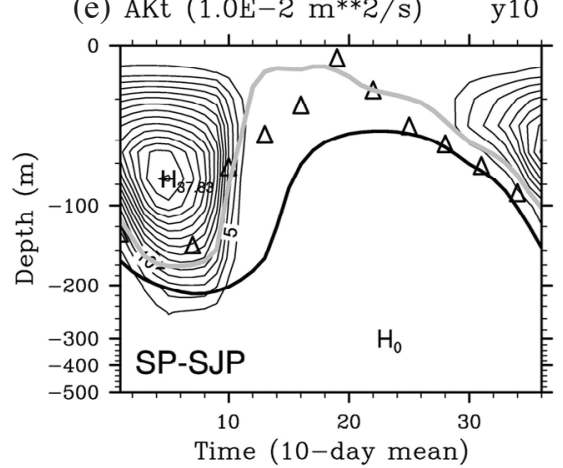

(c) $\operatorname{AKt}(1.0 \mathrm{E}-2 \mathrm{~m} * * 2 / \mathrm{s}) \quad \mathrm{y} 10$
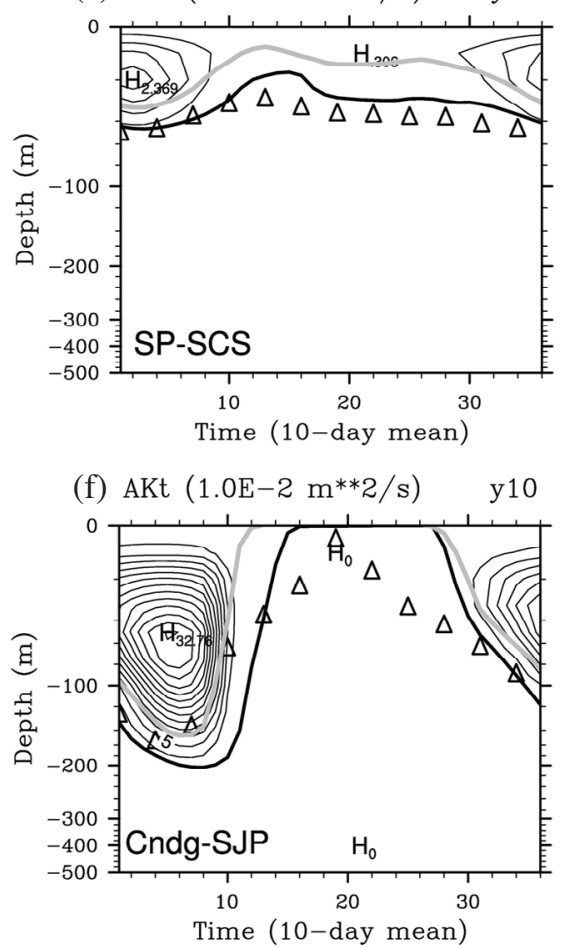

Fig. 14. The same as in Fig. 13, but for the boxed areas (a), (b) ETW, (c), (d) SCS, and (e), (f) SJP in experiments SP (left panels) and Cndg (right panels). 
too cold, as can be seen in Cndg and noSP (Fig. 10b). When the solar penetration is allowed, the summer heating occurs partly by the absorption of the radiation below the surface. The result is a reduction in the stability of the upper layer and increased mixing. The enhanced instability and mixing in the upper layers are expected to cause the deepening of the mixed layer.

\subsection{Upper Ocean Heat Budget}

In this section, the upper ocean heat budgets for SP and noFC are analyzed to understand the annual cycle of heat balance in the selected regions, and the difference in heat budget between the two experiments is analyzed to reveal the impact of the nudging terms (both for temperature and momentum nudging).

The tendency change of upper ocean heat content can be expressed by vertically integrated heat budget equation from sea surface to a fixed depth $(-h)$ :

$$
\begin{aligned}
\rho_{w} C_{p w} \int_{-h}^{0} \frac{\partial T}{\partial t} d z= & -\rho_{w} C_{p w} \int_{-h}^{0}\left(\vec{V} \cdot \nabla_{H} T+w \frac{\partial T}{\partial z}\right) d z \\
& +\rho_{w} C_{p w} \int_{-h}^{0} D_{T} d z+Q
\end{aligned}
$$

where $\nabla_{H}$ is the horizontal gradient operator. The rest of the notations are the same as in Eqs. (1) and (2). The term on the left-hand side of the equation is the local change of heat content (referred to as TEND). On the right-hand side, the first term is the heat advection (referred to as ADV), which consists of horizontal and vertical advections. In a large domain-mean budget, we may interpret ADV in terms of volume averaged flux divergence which is equivalent to the net flux convergence through the lateral and bottom walls. The second and last terms are the diffusive heat flux (referred to as DIF) and net surface heat flux (including the solar penetration; referred to as Qtot), respectively. The heat budget here is calculated for the top $400 \mathrm{~m}$ (or the water depth that is shallower than $400 \mathrm{~m}$ ) on each grid point. The choice of the depth $(400 \mathrm{~m})$ was based mainly on the reason that it is well below the thermocline such that the upper layer heat content change could be a reasonable indicative of the variability in the depth of the thermocline (and also the mixed layer depth). Furthermore, the vertical diffusive heat flux across this depth is expected to be small. In the four averaged regions, the horizontal and vertical diffusions were found to be sufficiently smaller than other terms, thus only the net effect of diffusive heat flux (DIF) will be presented below.

The annual cycle of the heat budget averaged in the four regions are shown in Fig. 15. We first describe similar features in the heat budget of SP and noFC (Figs. 15a, b). The local tendency term (TEND) shows a clear annual cycle with warming (cooling) in the warm (cool) season in each region. In the SCS, however, the warming period is shorter than others. Overall, the seasonality of the TEND can be attributed to the Qtot variation, especially in the cold season.

The major difference between the two experiments is found in the advection term (ADV) in $S J P$ and $E T W$ where the nudging term in noFC is quite large as revealed by the difference between TEND and SUM in Fig. 15b. The differences between SP and noFC are shown in Fig. 15c, where the differences in SUM are matched by the differences in ADV. This difference in ADV can be attributed to both the distributions of temperature (Figs. 8 and 10) and currents (Fig. 6). The large nudging term in the heat budget (note that there was also a momentum nudging in noFC but not shown here) indicates the model deficiency. This deficiency is restored in noFC by the nudging term, but leads to cooling in SP.

The above mentioned difference also exists in ECS and SCS but is much smaller so the overall budgets of the two experiments are in better agreement with each other. In the ECS and SCS, the relative importance of ADV and the surface heat flux show an interesting contrast. In SCS, the surface heat flux term is in phase with TEND in late winter and early spring, but the ADV term leads the flux term in summer. A reversed situation is observed in ECS where ADV leads the TEND in late winter and early spring but the surface flux term dominates in fall. The adjustments from the nudging terms are smaller in these two regions. Nevertheless, the differences in ADV again indicate the effect of heat advections are important mechanisms of local heat balance.

Without nudging, the TEND in SP is smaller than that in noFC. This is responsible for the cold bias in SP (Fig. 11). The annual averaged of the differences in TEND between SP and noFC are about $-20.8,-14.8,-8.6$, and $-13.0 \mathrm{~W} \mathrm{~m}^{-2}$ in $S J P, E T W, E C S$, and $S C S$, respectively. The cold biases in SP is likely caused by model deficiency, suggesting that the water column hydrodynamics has yet to reach a steady state and longer spinup time may be necessary. To address this issue warrants further investigation.

\subsection{Upper Ocean Transparency}

While the inclusion of solar penetration is critical for maintaining mixed layer depth in summer, the near-surface cold bias in SP (Fig. 10) may imply that the distribution of the penetrating solar radiation is inappropriate. The vertical distribution of the shortwave penetration is closely related to the ocean transparency to the solar radiation. In view of this, two additional experiments (SP3 and noFC.SP3) using a different water type, water type III (Jerlov 1976), is conducted to explore the sensitivity of the upper ocean thermal structure to different patterns of solar penetration. It is recalled that the water type III absorb more shortwave radiation in the top $2 \mathrm{~m}$, as compared with the water type I. The 
SP3 and noFC.SP3 are identical to SP and noFC, respectively, except the use of water type III.

The resultant temperature profiles are shown in Fig. 16. In all regions, the upper layer temperatures of SP3 are warmer than that of SP. In the winter, the larger tem- perature difference appears in the $E C S$ with a value at about $1^{\circ} \mathrm{C}$. In the summer, the temperature differences are about $2.5^{\circ} \mathrm{C}$ in the top $10 \mathrm{~m}$ in all regions. The upper layer temperature of SP3 in the ECS and SJP appear to tend towards the observation, while the temperature in the ETW and SCS (a) SP

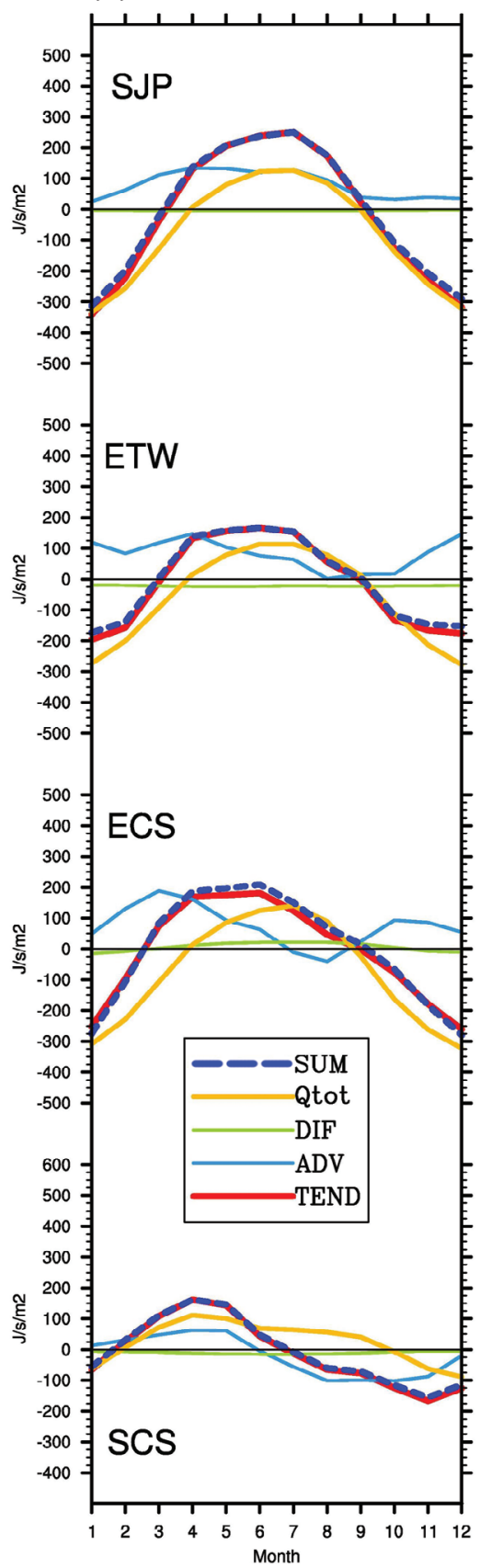

(b) noFC

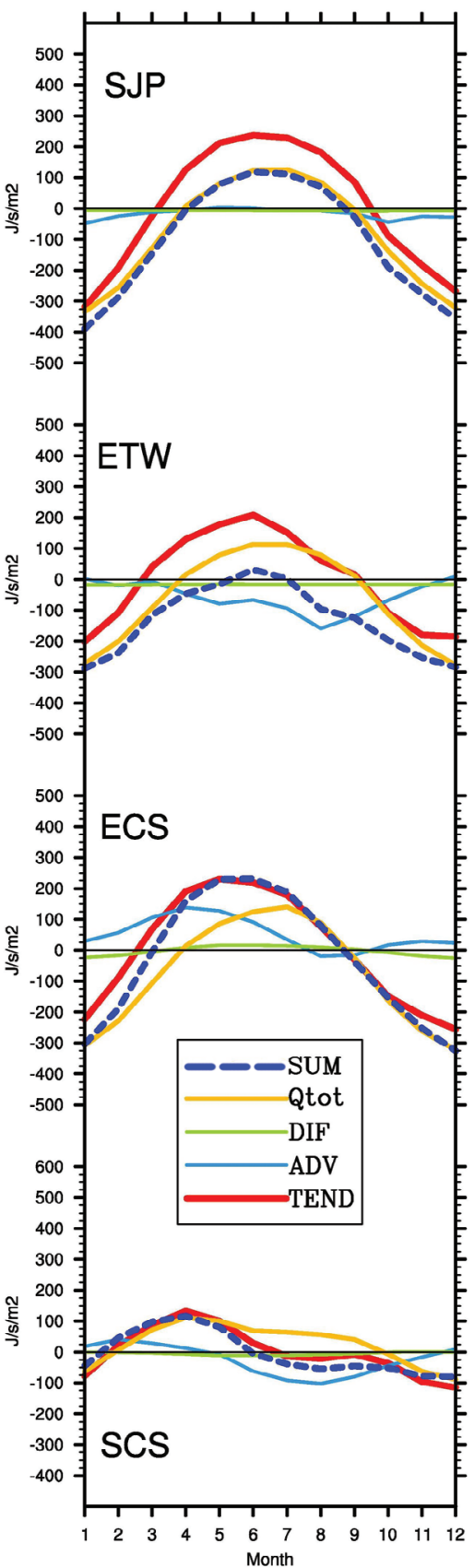

(c) SP-noFC

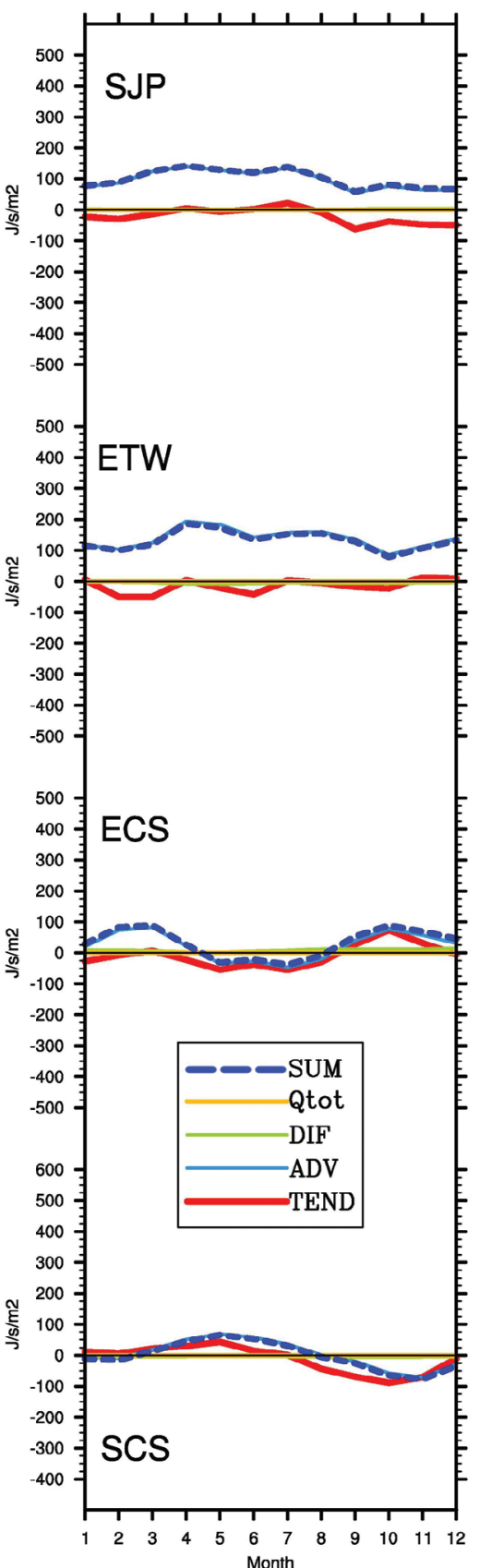

Fig. 15. Monthly mean of the terms in the heat content tendency equation for experiments (a) SP, (b) noFC, and (c) SP-noFC (difference between the two experiments). Term 'TEND' represents the local change of heat content. The terms 'ADV' and 'DIF' represent the combined contribution advections and diffusions, respectively. Term 'Qtot' is the net surface heat fluxes (includes the solar penetration). The term 'SUM' indicates the total contribution from the advections, diffusions, and surface heat fluxes. In noFC, the temperature nudging term can be expressed as the difference between TEND and SUM. The unit is $\mathrm{J} \mathrm{s}^{-1} \mathrm{~m}^{-2}\left(\right.$ also $\left.\mathrm{W} \mathrm{m}^{-2}\right)$. The monthly mean are calculated from the 5-year simulation of each experiment. The vertical integration is conducted from surface to a depth of $400 \mathrm{~m}$ (or the water depth, if it is shallower than $400 \mathrm{~m}$ ) on each grid point in regions $S J P, E T W, E C S$, and SCS. 
are warmer than the observation. Due to the higher upper layer temperature, the MLD in SP3 are slight shallower than that in SP. The use of water type III in noFC.SP3 also produces warmer upper layer temperature in summer, the temperatures differences are about $1 \sim 2^{\circ} \mathrm{C}$ in the top $10 \mathrm{~m}$. Consequently, summertime warm biases in the ECS, ETW, and $S C S$ are found in noFC.SP3. Proper use of the combination of solar penetration parameterization and temperature nudging coefficients is need.

In short, the simulation of the upper layer temperature is closely related to the ocean transparency, which is represented by the prescribed water type in our study. The experiments using the water type III, which allows more shortwave radiation to be absorbed near the sea surface, produce warmer near surface temperature and hence alleviate the cool biases significantly. However, the summertime warm biases in some regions, such as the ETW and SCS reveal that the use of a single water type may not be appropriate for a large domain as in our case. According to Martin (1985), the modeled MLD and surface temperatures are sensitive to the choice of the water type, which is a proxy of chlorophyll distribution (Jerlov 1976). The upper ocean solar irradiance penetration could vary significantly in response to westerly wind bursts in equatorial Pacific warm pool, which causes variation of water clarity in the upper ocean (Siegel et al. 1995). Large et al. (1994) also pointed out that simulations in spring and summer are sensitive to water clarity. An annual cycle of Jerlov (1976) water type is specified in their simulation for North Pacific, with clear type IA from December through February, type II from June through September, and type IB in the other months. To account for the lack of homogeneity of the ocean transparency, different water types with spatial and temporal variations may be prescribed in future works.

\subsection{Interannual Variability}

Three experiments are conducted to examine the performance of the model on interannual variability. These are the year_noFC, year_SP (Table 1), and year_SP3 which is

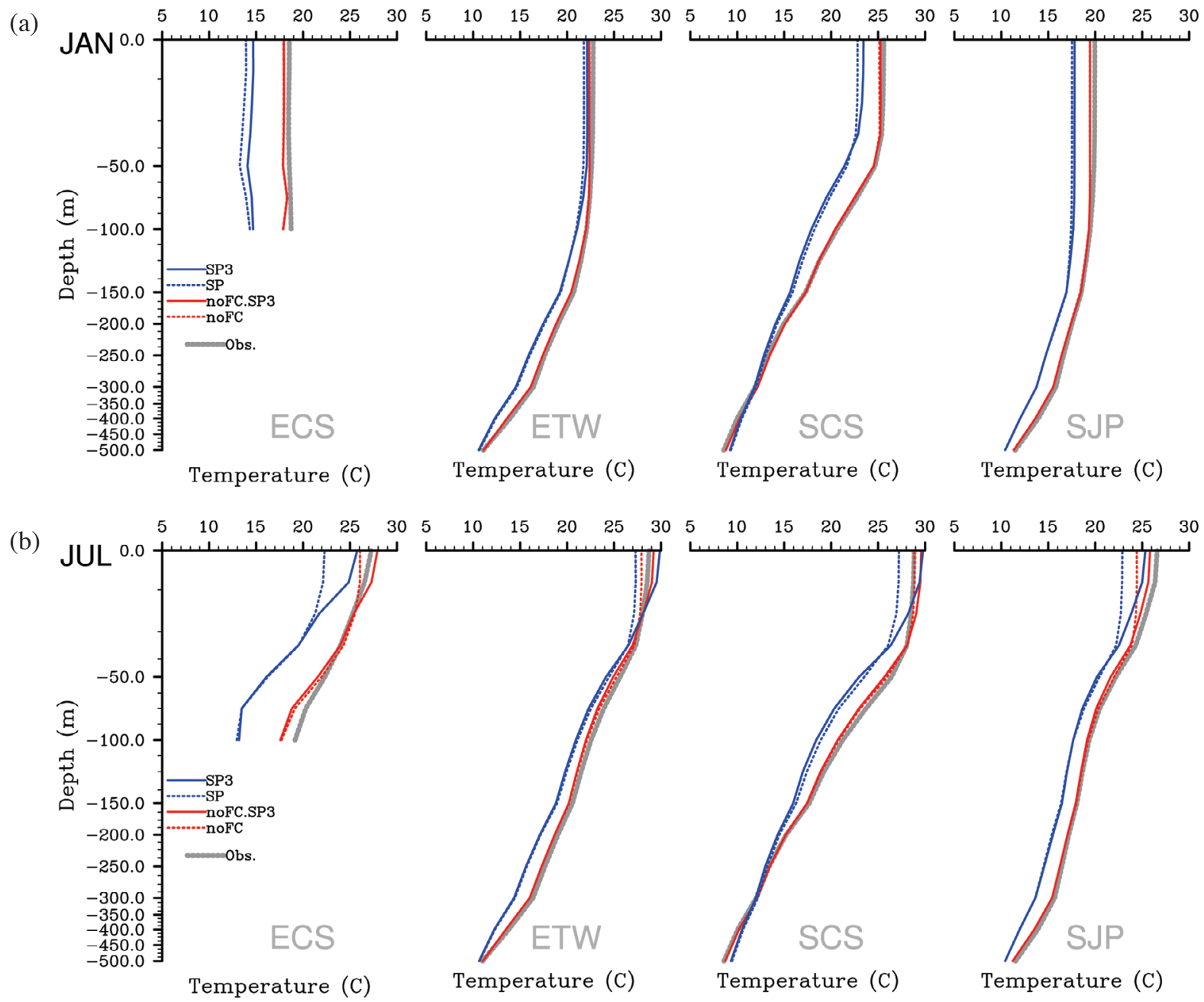

Fig. 16. The same as in Figure 10, but for the experiments noFC, noFC.SP3, SP, and SP3. 
identical to year_SP except for the use of water type III.

The time series of simulated SST and HC are presented in Fig. 17. The seasonality of the simulated SST is well reproduced. There are distinct annual cycles in all the regions except that in $S C S$, which exhibits quasi semi-annual cycle throughout the entire integration. The largest seasonal range appears in ECS, and the smallest occurs in SCS. The simulated SST in year_noFC is slight cooler than the observed SST derived from OISST. The cold bias of the simulated SST in year_SP is evident. The long-term drift of SST annual mean in the $E C S$ is up to $6.3^{\circ} \mathrm{C}$ after twelve years of integration. A larger departure is also found in SCS, with a difference approaching $4.4^{\circ} \mathrm{C}$ at the end of the simulation. The use of water type III in year_SP3 alleviates the summertime cool bias. The temporal evolutions of the simulated HC follow that of the simulated SST in all regions. Thus the simulated HC in year_SP and year_SP3 also exhibits significant long-term drift. The largest difference between the two experiments and the year_noFC appears in SJP.

The interannual variability of the simulated SST is examined in terms of the standardized anomaly. To remove the seasonal cycle in the SST anomaly, the monthly SST is subtracted from a long-term mean for that particular month. The long-term mean is defined as the model climatology for that month. The monthly anomaly is then divided by the standard deviation of that month to draw the standardized anomaly. Experiment year_noFC exhibits better correspondence than year_SP and year_SP3, in particular for the SST variations in 1995 - 2000 (Fig. 18). The linear correlation coefficients between the simulated SST from year_noFC and the observed SST are about 0.5 for the ETW, and about 0.4 for the ECS and SJP. Experiment year_SP3 produces higher correlation coefficients than year_SP in all regions, indicating a better representation of the ocean transparency on this time scale.

Overall, the interannual variability of $S S T$ is reasonably reproduced using a combination of the solar penetration and the nudging term in the integration. The result indicates that the nudging coefficient used in our study is still appropriate for the interannual simulation.

\section{DISCUSSION AND SUMMARY}

The ROMS model is adopted to develop a western North Pacific regional ocean model. A set of numerical experiments are carried out to explore the sensitivity of the model upper ocean thermal structure to some parameterized physical processes and correction schemes. The model results indicate that the solar penetration into the water column is quite important. The absorption of the solar radiation beneath the sea surface makes the upper layers less stable, increases mixing and thus deepens the MLD in the warm season. The stable upper layer in those experiments that do not have solar penetration results in overly shallow MLD in the warm season. This result is consistent with Martin (1985) and Ezer (2000). While the modeled MLD and surface temperatures are sensitive to the water clarity as found in previous studies (Martin 1985; Large et al. 1994; Ohlmann 2003; Sweeney et al. 2005), only single water types are used in our models to simplify discussion.

In addition to studying the effect of solar penetration on upper ocean thermal structure, a set of experiments are carried out to quantify the effect of flux correction and nudging terms on constraining the model from drifting away from more realistic thermal structure and flow field. In the absence of these constraints, the model forced by surface fluxes develops a cold bias. The heat budget analysis indicates that both heat advection and net surface heat flux are important terms for upper ocean heat balance in maintaining the climatological annual cycle. The heat advection, which consists of horizontal and vertical advections, is dependent on both currents and temperature fields. It is therefore sensitive to the model bias. Results from these experiments suggest that the cold bias is probably caused by too much heat dissipation at the open boundaries, while inadequate surface heat fluxes may be partially responsible as well. It is noteworthy that the net surface heat flux over the entire domain and the annual cycle is in deficit. While the use of water type III that allows more shortwave radiation to be absorbed near the surface tends to alleviate the summertime cool biases in some regions, it also exhibits warm biases in some other regions. Clearly, both the surface heat fluxes and the ocean transparency should be implemented carefully. Further investigation is warranted.

The alternative water type III use here can be use to represent an increase in solar irradiance attenuation within the upper water column, which can be attributed to an increase in the chlorophyll concentration. Lewis et al. (1990) indicated that variations in phytoplankton concentration in the equatorial Pacific is responsible for changes in heat trapped in the upper ocean. Siegel et al. (1995) also observed a significant reduction of solar penetration during a period of phytoplankton bloom in the western equatorial Pacific Ocean, which resulted in a chlorophyll concentration. To account for the influence of different light absorbing capability of the water column, different water types with spatial and temporal variations may be prescribed in future works. However, it is more desirable to couple the hydrodynamic model with a biogeochemical model such that a dynamic and interactive light absorbing profile could be generated to address this issue.

The flux correction approach appears effective in preventing the cold bias by adjusting the amounts of the net surface heat flux, but unrealistic net surface heat flux is produced in the experiment with unreasonable model assumption, such as the case without solar penetration into the upper water column. It is known that there are large uncertainties in specifying the correction coefficient in ocean 

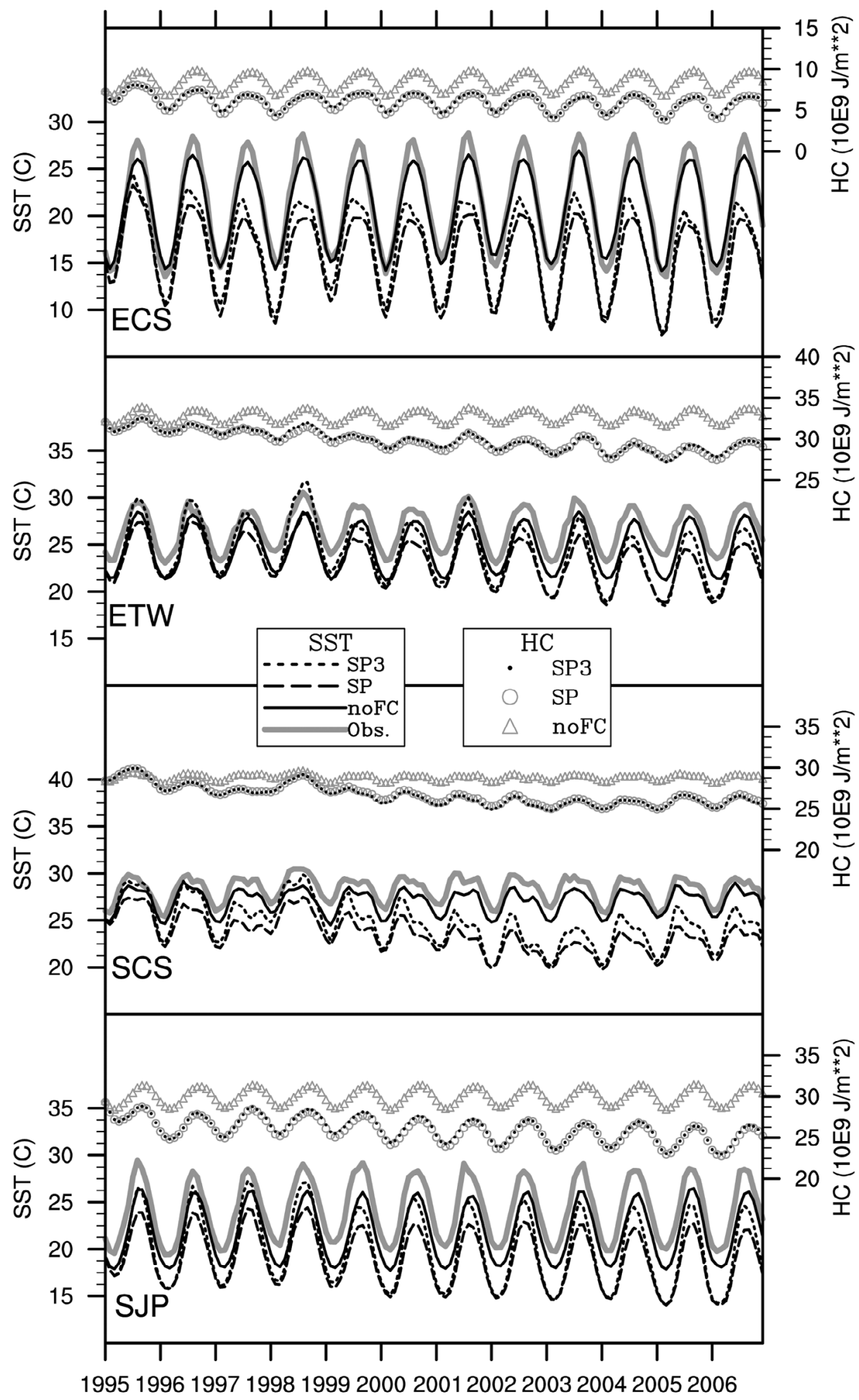

Time

Fig. 17. Time series of monthly mean SST (in Celsius) and heat content $\left(\mathrm{J} \mathrm{m}^{-2}\right)$ integrated for the upper $400 \mathrm{~m}$ over the regions ECS, ETW, SCS, and SJP for the interannual run from 1995 to 2006. The SST derived from experiments year_noFC, year_SP, and year_SP3 are indicated by solid, long-dashed and dashed lines, respectively, while the heat content for the three experiments are indicated by hollow triangles, hollow circles, and dots respectively. The SST from WOA98 monthly climatology is also plotted, and indicated by grey thick solid lines. 


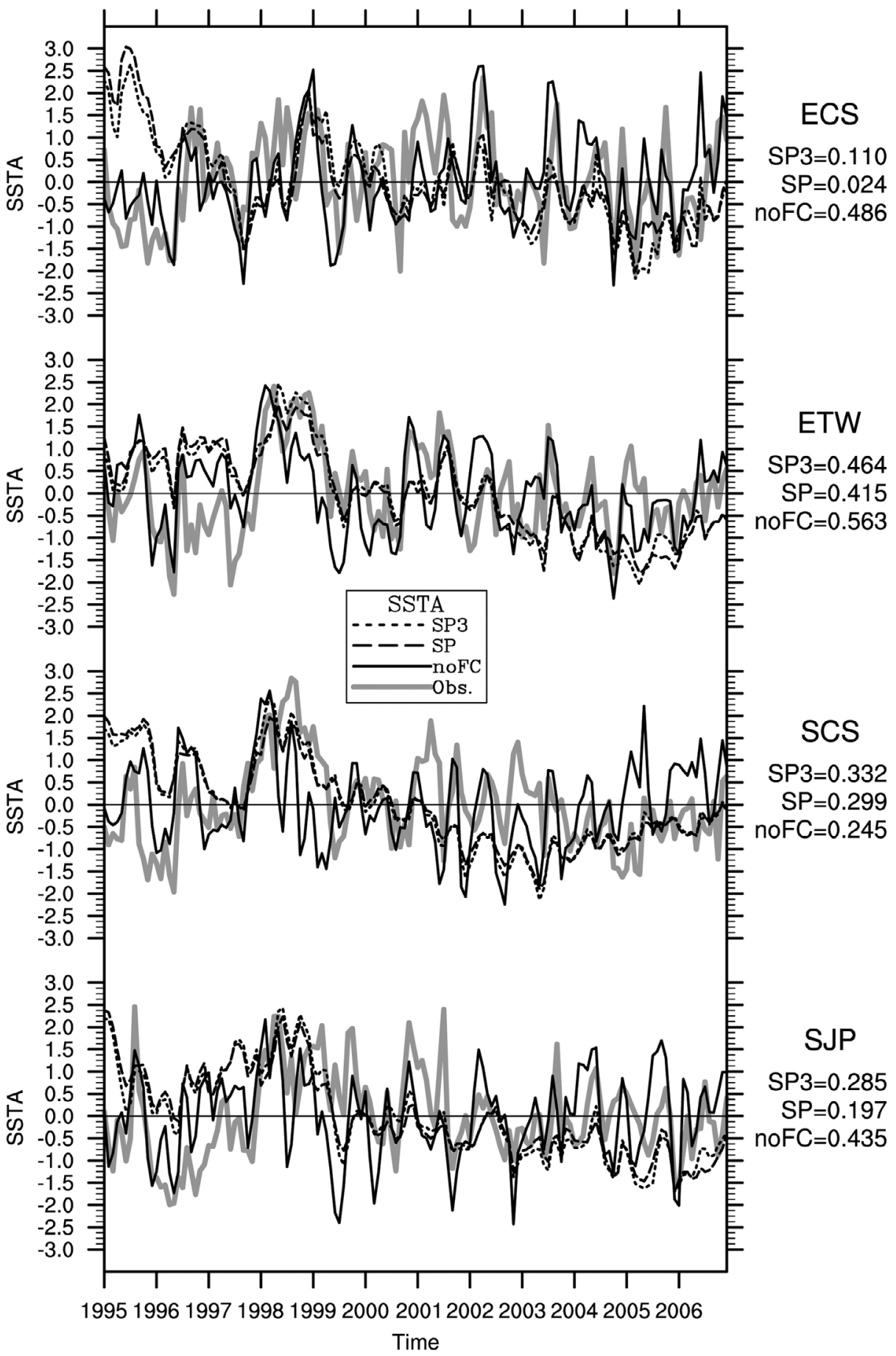

Fig. 18. Time series of standardized SST monthly anomalies over the regions (a) ECS, (b) ETW, (c) SCS, and (d) SJP for the interannual run from 1995 to 2006. The standardized SST monthly anomalies derived from experiments year_noFC, year_SP, and year_SP3 are indicated by solid, longdashed and dashed lines, respectively. The standardized SST monthly anomaly from WOA98 monthly climatology is also imposed, and indicated by grey thick solid lines. The linear correction coefficients between the three experiments and the WOA98 climatology are drawn.

modeling. Large et al. (1994) suggested that the values of SST difference should be less than $0.5^{\circ} \mathrm{C}$ every month for estimating the coefficient for a successful simulation of the North Pacific Ocean. Seager et al. (1995) mentioned that the sensitivity of the surface heat fluxes to variations in SST is much smaller than often assumed. They found that the flux response is strongly dependent on the scale of the SST anomaly. However, the magnitude of the scale dependent variation is only a few watts per square meter per Kelvin change of SST. This is less than half of the values typically 
assumed in ocean models. In addition to the uncertainty in the correction coefficient, the sensitivity of the upper ocean thermal structure to the flux correction process is also an open question. In his sensitivity study with a variety of the coefficients, Ezer (2000) found that the modeled MLD may be similar, but considerable differences in the heat content and SST may exist.

The implementation of a weak nudging toward the climatology prevents the model from a cold bias (drift from climatology) during long term integration. The nudging term seems to be able to account for some model biases, which may arise from model errors. The model errors may result from the spatial resolution or deficiency in the model forcing; for example, the absence of tidal forcing in our model. Furthermore, the variability in the model shall be constricted by the given nudging coefficient, which is often a timescale of several tens of days. The processes involving the nudging term remain unclear in our study. Nevertheless, the experiment with the solar penetration and the weak nudging produces reasonable interannual variability from 1995 to 2006 .

Acknowledgements We thank the two anonymous reviewers for their constructive comments. One of us (MTK) is grateful for the opportunity to study the ROMS model at the University of Maine and the support of the LORECS project. Special thanks are due to Dr. Katja Fennel, who provided useful information on setting up ROMS model. This study was supported by the National Science Council in Taiwan under Grants 95-2611-M-008 -005 and 96-2628M-008 -002.

\section{REFERENCES}

Barnett, T. P., D. W. Pierce, and R. Schnur, 2001: Detection of anthropogenic climate change in the world's ocean. Science, 292, 270-274, doi: 10.1126/science.1058304. [Link]

Beckmann, A. and D. B. Haidvogel, 1993: Numerical simulation of flow around a tall isolated seamount. Part I: Problem formulation and model accuracy. J. Phys. Oceanogr., 23, 1736-1753, doi: 10.1175/1520-0485(1 993)023<1736:NSOFAA $>2.0 . C O ; 2$. [Link]

Carton, J. A., G. Chepurin, X. Cao, and B. Giese, 2000a: A Simple Ocean Data Assimilation analysis of the global upper ocean 1950-1995. Part 1: Methodology. J. Phys. Oceanogr., 30, 294-309, doi: 10.1175/1520-0485(200 0)030<0294:ASODAA >2.0.CO;2. [Link]

Carton, J. A., G. Chepurin, and X. Cao, 2000b: A Simple Ocean Data Assimilation analysis of the global upper ocean 1950-1995 Part 2: Results. J. Phys. Oceanogr., 30, 311-326, doi: 10.1175/1520-0485(2000)030 $<0311$ :ASODAA > 2.0.CO;2. [Link]

da Silva, A. M., C. C. Young, and S. Levitus, 1994: Atlas of surface marine data 1994, Vol. 1, Algorithms and Pro- cedures, Technical Report, US Department of Commerce, NOAA, 1994.

Ezer, T., 2000: On the seasonal mixed layer simulated by a basin-scale ocean model and the Mellor-Yamada turbulence scheme. J. Geophys. Res., 105, 16843-16855, doi: 10.1029/2000JC900088. [Link]

Guilyardi, E., A. Wittenberg, A. Fedorov, M. Collins, C. Wang, A. Capotondi, G. J. van Oldenborgh, and T. Stockdale, 2009: Understanding El Niño in oceanatmosphere general circulation models: Progress and challenges. Bull. Amer. Meteorol. Soc., 90, 325-340, doi: 10.1175/2008BAMS2387.1. [Link]

Haidvogel, D. B., H.G. Arango, K. Hedstrom, A. Beckmann, P. Malanotte-Rizzoli, and A. F. Shchepetkin, 2000: Model evaluation experiments in the north Atlantic basin: Simulations in nonlinear terrain-following coordinates. Dyn. Atmos. Oceans, 32, 239-281, doi: 10.1016/S0377-0265(00)00049-X. [Link]

Haidvogel, D. B., H. Arango, W. P. Budgell, B. D. Cornuelle, E. Curchitser, E. Di Lorenzo, K. Fennel, W. R. Geyer, A. J. Hermann, L. Lanerolle, J. Levin, J. C. McWilliams, A. J. Miller, A. M. Moore, T. M. Powell, A. F. Shchepetkin, C. R. Sherwood, R. P. Signell, J. C. Warner, and J. Wilkin, 2008: Ocean forecasting in terrain-following coordinates: Formulation and skill assessment of the Regional Ocean Modeling System. J. Comput. Phys., 227, 3595-3624, doi: 10.1016/j.jcp. 2007.06.016. [Link]

Haney, R. L., 1971: Surface thermal boundary condition for ocean circulation models. J. Phys. Oceanogr., 1, 241-248, doi: 10.1175/1520-0485(1971)001<0241:ST $\mathrm{BCFO}>2.0 . \mathrm{CO}$;2. [Link]

Hansen, J., L. Nazarenko, R. Ruedy, M. Sota, J. Willis, A. D. Genio, D. Koch, A. Lacis, K. Lo, S. Menon, T. Novakov, J. Perlwitz, G. Russell, G. A. Schmidt, and N. Tausnev, 2005: Earth's energy imbalance: Confirmation and implications. Science, 308, 1431-1435, doi: 10.1126/science.1110252. [Link]

Jan, S., D. D. Sheu, and H. M. Kuo, 2006: Water mass and throughflow transport variability in the Taiwan Strait. J. Geophys. Res., 111, C12012, doi: 10.1029/ 2006JC003656. [Link]

Jerlov, N. G., 1976: Marine Optics, Elsevier, New York, $231 \mathrm{pp}$.

Kagimoto, T. and T. Yamagata, 1997: Seasonal transport variations of the Kuroshio: An OGCM simulation. $J$. Phys. Oceanogr., 27, 403-418, doi: 10.1175/1520-0485 (1997)027<0403:STVOTK>2.0.CO;2. [Link]

Large, W. G., J. C. McWilliams, and S. C. Doney, 1994: Oceanic vertical mixing: A review and a model with a nonlocal boundary layer parameterization. Rev. Geophys., 32, 363-403.

Levitus, S., J. Antonov, T. P. Boyer, and C. Stephens, 2000: Warming of the world ocean. Science, 287, 2225-2229, 
doi: 10.1126/science.287.5461.2225. [Link]

Levitus, S., J. Antonov, and T. P. Boyer, 2005: Warming of the world ocean, 1955-2003. Geophys. Res. Lett., 32, L02604, doi: 10.1029/2004GL021592. [Link]

Lewis, M. R., M. E. Carr, G. C. Feldman, W. Esaias, and C. McClain, 1990: Influence of penetrating solar radiation on the heat budget of the equatorial Pacific Ocean. Nature, 347, 543-545, doi: 10.1038/347543a0. [Link]

Marotzke, J. and J. Willebrand, 1991: Multiple equilibria of the global thermohaline circulation. J. Phys. Oceanogr., 21, 1372-1385, doi: 10.1175/1520-0485(1991)021<1372:MEOTGT>2.0.CO;2. [Link]

Martin, P. J., 1985: Simulation of the mixed layer at OWS November and Papa with several models. J. Geophys. Res., 90, 903-916. [Link]

Oey, L. Y. and P. Chen, 1992: A model simulation of circulation in the northeast Atlantic shelves and seas. $J$. Geophys. Res., 97, 20087-20115. [Link]

Ohlmann, J. C., 2003: Ocean radiant heating in climate models. J. Climate, 16, 1337-1351, doi: 10.1175/15200442(2003)16<1337:ORHICM>2.0.CO;2. [Link]

Paulson, C. A. and J. J. Simpson, 1977: Irradiance measurements in the upper ocean. J. Phys. Oceanogr., 7, 952956, doi: 10.1175/1520-0485(1977)007<0952:IMIT $\mathrm{UO}>2.0 . \mathrm{CO} ; 2$. [Link]

Pielke, Sr., R. A., 2003: Heat storage within the Earth system. Bull. Amer. Meteorol. Soc., 84, 331-335, doi: 10.1175/BAMS-84-3-331. [Link]

Qu, T., 2000: Upper-layer circulation in the South China Sea. J. Phys. Oceanogr., 30, 1450-1460.

Qu, T. and R. Lukas, 2003: The bifurcation of the north equatorial current in the Pacific. J. Phys. Oceanogr., 33, 5-18.

Qu, T., Y. Du, J. Gan, and D. Wang, 2007: Mean seasonal cycle of isothermal depth in the South China Sea. J. Geophys. Res., 112, C02020, doi: 10.1029/2006JC003583. [Link]

Qu, T., J. Gan, A. Ishida, Y. Kashino, and T. Tozuka, 2008: Semiannual variation in the western tropical Pacific Ocean, Geophys. Res. Lett., 35, L16602, doi: 10.1029/2008GL035058. [Link]

Reynolds, R. W. and T. M. Smith, 1994: Improved global sea surface temperature analyses. J. Climate, 7, 929-948, doi: 10.1175/1520-0442(1994)007<0929:IG SSTA $>2.0 . C O ; 2$. [Link]

Reynolds, R. W., N. A. Rayner, T. M. Smith, D. C.
Stokes, and W. Wang, 2002: An improved in situ and satellite SST analysis for climate. J. Climate, 15, 1609-1625, doi: 10.1175/1520-0442(2002)015<1609: AIISAS > 2.0.CO;2. [Link]

Rossby, C., 1959: Current problems in meteorology. In: Bolin, B. (Ed.), the Atmosphere and Sea in Motion, Rockefeller Inst. Press, New York, 9-50.

Sarmiento, J. L. and K. Bryan, 1982: An ocean transport model for the North Atlantic. J. Geophys. Res., 87, 394-408, doi: 10.1029/JC087iC01p00394. [Link]

Seager, R., Y. Kushnir, and M. A. Cane, 1995: On heat flux boundary conditions for ocean models. J. Phys. Oceanogr., 25, 3219-3230, doi: 10.1175/1520-0485(1995)025<3219:OHFBCF>2.0.CO;2. [Link]

Shchepetkin, A. F. and J. C. McWilliams, 1998: Quasimonotone advection schemes based on explicit locally adaptive dissipation. Mon. Weather Rev., 126, 15411580, doi: 10.1175/1520-0493(1998)126<1541:QMA $\mathrm{SBO}>2.0 . \mathrm{CO} ; 2$. [Link]

Shchepetkin, A. F. and J. C. McWilliams, 2003: A method for computing horizontal pressure-gradient force in an oceanic model with a non-aligned vertical coordinate. $J$. Geophys. Res., 108, 1-34, doi: 10.1029/2001JC001047. [Link]

Siegel, D. A., J. C. Ohlmann, L. Washburn, R. R. Bidigare, C. T. Nosse, E. Fields, and Y. M. Zhou, 1995: Solarradiation, phytoplankton pigments and the radiant heating of the Equatorial Pacific Warm Pool. J. Geophys. Res., 100, 4885-4891, doi: 10.1029/94JC03128. [Link]

Song, Y. and D. B. Haidvogel, 1994: A semi-implicit ocean circulation model using a generalized topographyfollowing coordinate. J. Comput. Phys., 115, 228-244, doi: 10.1006/jcph.1994.1189. [Link]

Sweeney, C., A. Gnanadesikan, S. M. Griffies, M. J. Harrison, A. J. Rosati, and B. L. Samuels, 2005: Impacts of shortwave penetration depth on large-scale ocean circulation and heat transport. J. Phys. Oceanogr., 35, 1103-1119, doi: 10.1175/JPO2740.1. [Link]

Weaver, A. J. and E. S. Sarachik, 1991: Evidence for decadal variability in an ocean general circulation model: An advective mechanism. Atmos. Ocean, 29, 197-231.

Xue, H., F. Chai, N. Pettigrew, D. Xu, M. Shi, and J. Xu, 2004: Kuroshio intrusion and the circulation in the South China Sea. J. Geophys. Res., 109, C02017, doi: 10.1029/2002JC001724. [Link] 